\title{
Validated and longitudinally stable asthma phenotypes based on cluster analysis of the ADEPT study
}

Matthew J. Loza' ${ }^{1}$, Ratko Djukanovic ${ }^{2}$, Kian Fan Chung ${ }^{3}$, Daniel Horowitz ${ }^{1}$, Keying Ma ${ }^{1}$, Patrick Branigan', Elliot S. Barnathan ${ }^{1}$, Vedrana S. Susulic ${ }^{1}$, Philip E. Silkoff ${ }^{1}$, Peter J. Sterk ${ }^{4}$, Frédéric Baribaud ${ }^{1 *}$ (D) and For the ADEPT (Airways Disease Endotyping for Personalized Therapeutics) and U-BIOPRED (Unbiased Biomarkers for the Prediction of Respiratory Disease Outcome Consortium) investigators

\begin{abstract}
Background: Asthma is a disease of varying severity and differing disease mechanisms. To date, studies aimed at stratifying asthma into clinically useful phenotypes have produced a number of phenotypes that have yet to be assessed for stability and to be validated in independent cohorts. The aim of this study was to define and validate, for the first time ever, clinically driven asthma phenotypes using two independent, severe asthma cohorts: ADEPT and U-BIOPRED.

Methods: Fuzzy partition-around-medoid clustering was performed on pre-specified data from the ADEPT participants $(n=156)$ and independently on data from a subset of U-BIOPRED asthma participants $(n=82)$ for whom the same variables were available. Models for cluster classification probabilities were derived and applied to the 12-month longitudinal ADEPT data and to a larger subset of the U-BIOPRED asthma dataset $(n=397)$. High and low type-2 inflammation phenotypes were defined as high or low Th2 activity, indicated by endobronchial biopsies gene expression changes downstream of IL-4 or IL-13.

Results: Four phenotypes were identified in the ADEPT (training) cohort, with distinct clinical and biomarker profiles. Phenotype 1 was "mild, good lung function, early onset", with a low-inflammatory, predominantly Type-2, phenotype. Phenotype 2 had a "moderate, hyper-responsive, eosinophilic" phenotype, with moderate asthma control, mild airflow obstruction and predominant Type-2 inflammation. Phenotype 3 had a "mixed severity, predominantly fixed obstructive, non-eosinophilic and neutrophilic" phenotype, with moderate asthma control and low Type-2 inflammation. Phenotype 4 had a "severe uncontrolled, severe reversible obstruction, mixed granulocytic" phenotype, with moderate Type-2 inflammation. These phenotypes had good longitudinal stability in the ADEPT cohort. They were reproduced and demonstrated high classification probability in two subsets of the U-BIOPRED asthma cohort.
\end{abstract}

Conclusions: Focusing on the biology of the four clinical independently-validated easy-to-assess ADEPT asthma phenotypes will help understanding the unmet need and will aid in developing tailored therapies.

Trial registration: NCT01274507 (ADEPT), registered October 28, 2010 and NCT01982162 (U-BIOPRED), registered October 30, 2013.

Keywords: Cluster analysis, Biological markers, Observational study

\footnotetext{
* Correspondence: fbaribau@its.jnj.com

${ }^{1}$ Immunology Therapeutic Area, Janssen Research \& Development LLC, 1400

McKean Rd, Spring House 19477, USA

Full list of author information is available at the end of the article
} 


\section{Background}

Asthma is a disease driven by complex and heterogeneous pathobiologic processes, involving a multitude of inflammatory and structural cell types and a large number of pro-inflammatory and tissue remodeling mediators [1]. This heterogeneity, may explain, at least in part, the severity of the disease, including varying risk of exacerbations [2] and the inconsistency in responses observed across the spectrum of asthma patients to both standard therapies and the emerging biologics $[3,4]$.

Despite a plethora of published studies on asthma mechanisms, the definition of asthma remains limited to the description of its key clinical features, with broad reference to the underlying inflammatory characteristics and heterogeneity. This is true for both general definitions provided by the Global Initiative for Asthma guidelines (GINA, http://www.ginasthma.org/) and definitions of its severe forms [1], while stratification of asthma is still based on the combination of symptoms, lung function and treatment required for symptom control. Recognizing that clinical and pathobiologic features do not follow a linear, incremental pattern presented in the GINA guidelines, a number of studies of asthmatics with varying clinical presentations have applied unbiased clustering in an attempt to define new phenotypes of asthma. These studies have used a range of clinical variables in combination with simple, easy to apply, measures of airways inflammation assessed in induced sputum [5-8]. Indeed, the Severe Asthma Research Program (SARP) clustered on clinical, demographic, and natural history variables from 726 asthmatics, resulting in 5 patient clusters [5]. Subsequently, in a subset of these subjects $(n=423), 15$ inflammatory cellular measures and clinical variables were included, and 4 clusters were identified [6]. In a recent SARP report, the dataset was reduced $(n=378)$ to participants with exhaled nitric oxide (FENO) and bronchoalveolar lavage (BAL) fluid cell counts, and included healthy controls, resulting in 6 clusters by clustering on 112 clinical, physiologic, and inflammatory variables [7]. Patient data from the Dose Ranging Efficacy And safety with Mepolizumab (DREAM) study was clustered on clinical and biomarker variables [8]. Four clusters were identified that could be defined by 3 predictors (blood eosinophils, airway reversibility, and body mass index). However, neither the longitudinal stability of the described clusters nor their validation in an independent asthma cohort has reported to date. Furthermore, limited studies to date have undertaken an in-depth characterization of the molecular processes that are associated with the clinical phenotypes. Such analysis is limited to a single study of mild, steroidnaive asthmatics by Woodruff and colleagues [9] who assessed gene transcription in the airways of 42 nonsmoking subjects with asthma, 28 nonsmoking healthy controls, and 16 current smokers without asthma but with mild to moderate airflow obstruction (disease controls) and observed two broad clusters defined by the expression of three genes (POSTN, CLCA1 and SERPINB2) induced by the type-2 (T2) cytokine interleukin (IL)-13. The two clusters, defined by high and low expressions levels of these three genes, were termed Th2-high and Th2-low, the latter composed of asthmatic and healthy individuals.

The current study had two main aims. The first aim was to identify clinical phenotypes defined by a limited set of clinical variables that are easily acquired and can, therefore, be used in routine clinical practice or trials, and to assess their reproducibility and stability over time. The second aim was to describe these clinical phenotypes by their molecular characteristics, as assessed by whole genome expression of bronchial samples acquired by bronchoscopic biopsies and brushings. The study used clinical and biomarker data from two independent cohorts. Initial clustering that created the clinical phenotypes was performed on clinical data acquired in the ADEPT (Airway Disease Endotyping for Personalized Therapeutics) study [10] involving 158 asthmatics. The phenotypes thereby produced were assessed for stability within the longitudinal arm of the ADEPT study and validated for reproducibility using data from a subset of asthmatics from the U-BIOPRED (Unbiased Biomarkers for the Prediction of Respiratory Disease Outcome) study [11] constituting an independent validation cohort.

\section{Methods}

\section{Study design and study populations}

The ADEPT and U-BIOPRED study designs and study populations are published elsewhere $[10,11]$ and are described briefly here. The current study consisted of a combination of cross-sectional (ADEPT and U-BIOPRED) and longitudinal (ADEPT) studies. Participants in both cohorts were assessed clinically at baseline, using prespecified protocols.

Clinical phenotypes were created by clustering 9 clinical variables (see Clustering methodology in the next paragraph) from the ADEPT study which served as the training set. Matching datasets from U-BIOPRED, excluding airway hyperresponsiveness (AHR), which was not assessed in U-BIOPRED, were used as the validation set for the ADEPT study-derived clinical phenotypes. After 3, 6 and 12 months of follow up, the ADEPT study participants were further reassessed to enable further validation, i.e. assessment of the stability of the phenotypes through classification of the followed up participants into the ADEPT study-derived phenotypes created with baseline data. Relevant biological samples were collected to define the pathobiologic (transcriptomic) characteristics of the clinical phenotypes. Both the ADEPT [10] and U-BIOPRED [11] studies collected serum for protein array analysis. All participants underwent 
bronchoscopy for differential gene expression analysis by microarray of endobronchial biopsies (in ADEPT) and epithelial brushings (U-BIOPRED) (reasons for using different sample types are described below under "Airway type-2 inflammation high and low phenotype"). All ADEPT participants underwent sputum induction at the screening visit, to fulfil inclusion criteria, and again at the Baseline Visit, to confirm the stability of their phenotype, while the U-BIOPRED participants had a single sputum induction.

Detailed inclusion and initial clinical stratification criteria of the two studies are published elsewhere $[10,11]$. Briefly, for recruitment purposes, the 158 asthmatic participants in the ADEPT cohort were initially classified into severity groups based on their use of asthma controller medication and lung function as assessed by forced expiratory volume in one second $\left(\mathrm{FEV}_{1}\right)$ : mild (no asthma controller medications, $\mathrm{FEV}_{1}>80 \%$ of predicted) $(n=52)$, moderate (low-moderate dose ICS, FEV 160 $80 \%$ of predicted) $(n=55)$, or severe (on high-dose ICS, $\mathrm{FEV}_{1} 50-80 \%$ of predicted) $(n=51) . \mathrm{FEV}_{1}$ ranges were selected to reflect appropriate medication level (e.g., if a patient had $\mathrm{FEV}_{1}<60 \%$ of predicted, a low-medium ICS dose may not be appropriate), with a low-bound in the severe group for safety considerations. All had been current non-smokers for at least one year, with $<10$ packyear smoking history. The 530 asthmatics from the UBIOPRED cohort were initially classified as non-severe (low-medium dose ICS) $(n=88)$, non-smoking severe (high-dose ICS; current non-smokers with $<5$ packyear smoking history) $(n=110)$, and smoking severe (high-dose ICS; current or ex-smokers with at least 5 pack-year smoking history) $(n=311)$. All the asthmatics on maintenance oral corticosteroids (OCS) in the UBIOPRED cohort were classified as severe. A subset of the U-BIOPRED cohort only was included in this study using those participants without missing data for the 8 clustering variables described below $(n=397$ of 509) (see Additional file 1: Table S2). The 397 U-BIOPRED participants included both participants on maintenance OCS and smokers, two groups of participants which were not represented in ADEPT.

Clinical phenotypes were created by clustering 9 clinical variables (see Clustering methodology in the next paragraph) from the ADEPT study which served as the training set. Matching datasets from U-BIOPRED, excluding airway hyperresponsiveness (AHR), which was not assessed in U-BIOPRED, were used as the validation set for the ADEPT study-derived clinical phenotypes. After 3, 6 and 12 months of follow up, the ADEPT study participants were further reassessed to enable further validation, i.e. assessment of the stability of the phenotypes through classification of the followed up participants into the ADEPT study-derived phenotypes created with baseline data. Relevant biological samples were collected to define the pathobiologic (transcriptomic) characteristics of the clinical phenotypes. Both the ADEPT [10] and UBIOPRED [11] studies collected serum for protein array analysis. All participants underwent bronchoscopy for differential gene expression analysis by microarray of endobronchial biopsies (in ADEPT) and epithelial brushings (U-BIOPRED) (reasons for using different sample types are described below under "Airway type- 2 inflammation high and low phenotype". All ADEPT participants underwent sputum induction at the screening visit, to fulfil inclusion criteria, and again at the Baseline Visit, to confirm the stability of their phenotype, while the U-BIOPRED participants had a single sputum induction.

\section{Clustering methodology}

Figure 1 describes the overall schematic of clustering analyses. Initial clustering of the clinical data from the independent ADEPT (\#156 of 158) and U-BIOPRED (\#82 of 509) cohorts was performed using the Fuzzy Partition-around-Medoid (PAM) clustering method [12] to create the baseline phenotypes. For the purpose of validation of the identified phenotypes, the GLMnetclassification model of ADEPT-asthma baseline clinical clusters (\#154 of 158) was then applied to classify the ADEPT-asthma subjects using the data from the baseline and 3, 6, and 12 month follow-up visits and the baseline data of U-BIOPRED participants (\#397 of 509). The discrepancies between the cohort sizes and the number of patients used were due to missing data for various clustering variables.

Nine clustering variables were selected because they can be readily measured in standard clinics: pre-bronchodilator forced expiratory volume in $1 \mathrm{~s}$ (pre-BD $\mathrm{FEV}_{1}$ ) and forced vital capacity (FVC) expressed as \% predicted, FEV1/FVC ratio, bronchodilator reversibility (BDR), airway hyperresponsiveness (AHR), i.e. log-transformed provocative concentration of methacholine resulting in 20\% decline in $\mathrm{FEV}_{1}$ from baseline $\left(\mathrm{PC}_{20}\right)$; Asthma Control Questionnaire (ACQ-7) [13], Asthma Quality of Life Questionnaire (AQLQ) [14]; log-transformed Fractional Exhaled Nitric Oxide (FENO) concentration in exhaled breath, and blood eosinophil counts expressed as absolute counts per $\mu \mathrm{L}$.

PAM clustering is moderately robust to missing data, allowing the PC20 variable to be utilized despite a minority of ADEPT-asthma patients (20 of 158) not having values for this variable (methacholine challenge was not performed if FEV1 was $<60 \%$ predicted). Two subjects in the mild asthma cohort were excluded because they did not have valid screening or baseline pre-bronchodilator spirometry measurements available, resulting in not having values for 4 of the 9 variables. Two ADEPT asthma subjects were missing baseline data for FENO and were included in the initial clustering but excluded from 


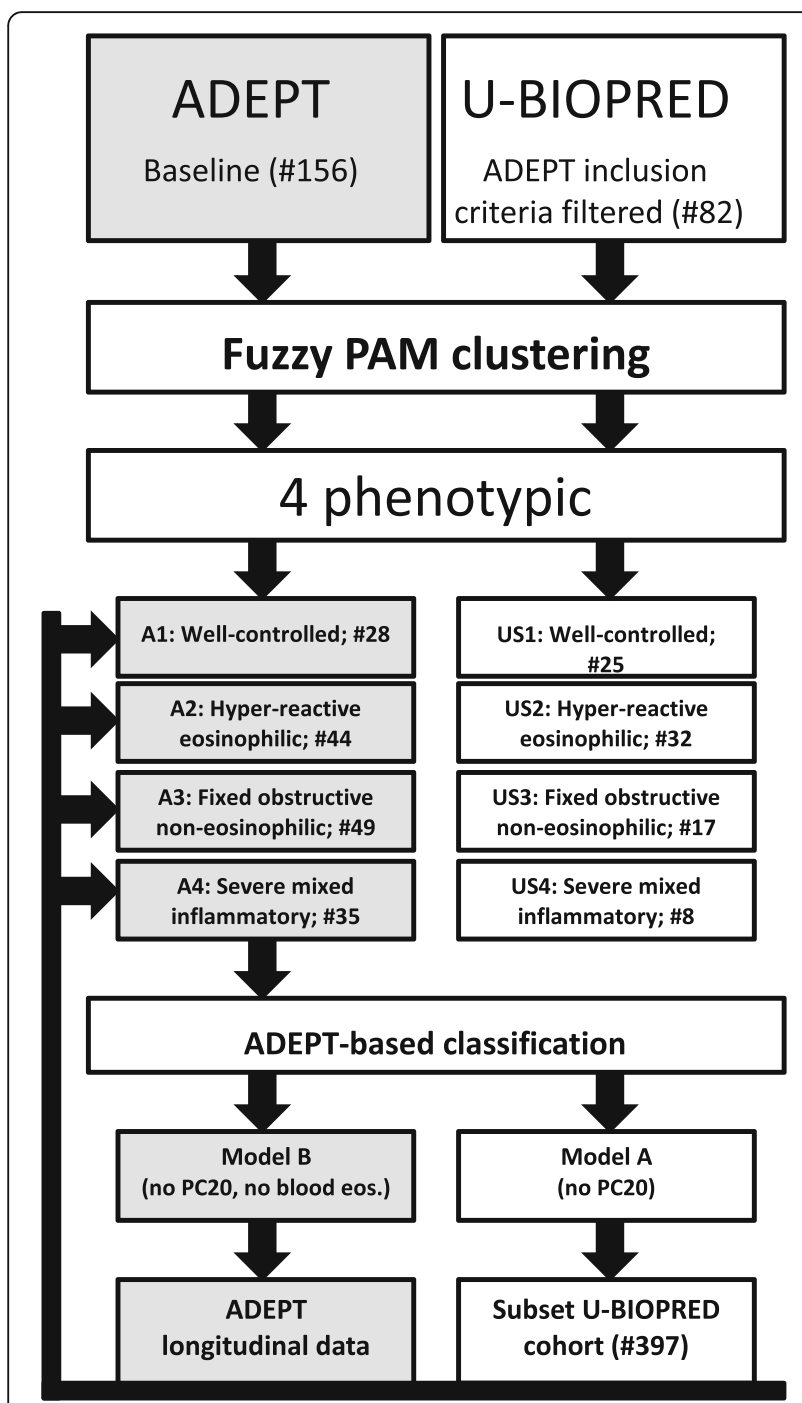

Fig. 1 Schematic of clustering analyses. Fuzzy PAM clustering was used on 156 ADEPT and 82 U-BIOPRED asthma patients, defining analogous phenotypes, A1 to A4 for ADEPT and US1 to US4 for U-BIOPRED. GLMnet classification models for the ADEPT phenotypes was built and applied to either the ADEPT longitudinal samples (3,6 and 12 month) or a large subset of the U-BIOPRED cohort $(n=397)$

GLMnet classification analyses, described below. Therefore, $156 \mathrm{ADEPT}$ asthma subjects were included in the initial clustering, and 154 for GLMnet classification analyses.

\section{Initial clustering}

The Fuzzy Partition-around-Medoid (PAM) clustering method [12] (software: NCSS v8, www.NCSS.com; NCSS LLC, Kaysville, Utah) used Euclidean distance, scaled with average absolute deviation, and applied the 'fuzzifier constant' set to 1.1 (value selected to optimize Silhouette and distance metrics). The number of clusters selected for further analysis was based on maximizing between cluster distance (normalized Dunn's partition coefficient, $(\mathrm{Fc}(\mathrm{U}))$ and minimizing within-cluster distance (normalized Kaufman's distance partition coefficient, $(\mathrm{Dc}(\mathrm{U})))$ metrics [12]. The fuzzy clustering algorithm assigns to each subject a probability of membership to each of the clusters, with the subject then assigned to the cluster with the highest probability of membership.

Fuzzy PAM was selected because it is robust to outliers and missing data, does not make the assumption that all participants cleanly belong to a single cluster and because it has advantages over other standard methods, such as k-means clustering and hierarchical clusters, as well as traditional PAM. Because of limited sample sizes, permutation-based methods to identify stable clusters were not employed. Sensitivity analyses were performed to assess the robustness of these initial clusters in ADEPT (removing the medoid subjects and whole study arms), the longitudinal stability of the clusters, and the homologous clusters observed when clustering on U-BIOPRED subjects, overcome the limitations of not employing permutations.

For clustering of ADEPT participants, all 158 asthmatics from the mild, moderate, and severe study arms were included, with 2 mild asthmatics excluded because they did not have valid screening or baseline pre-bronchodilator spirometry measurements available. For clustering of U-BIOPRED participants, clustering was performed using the same methods and variables as ADEPT but excluding PC20 because this variable was available only in a minority of UBIOPRED subjects. Participants with missing data for any of the 8 clustering variables or those taking maintenance OCS were also excluded to ensure the most robust clustering ( $n=82$ of 509 total participants, with additional exclusion of 194 on maintenance OCS, 64 smoking severe asthmatics, 148 with $\mathrm{FEV}_{1}$ out-of-range, and additional 21 with missing data).

\section{GLMnet classification}

The GLMNet multinomial logistic regression classification algorithm ( $\mathrm{R}$ package GLMNet v1.9-8 7 [15], http://www.jstatsoft.org/v33/i01/) was used with the R-package Caret (Classification and Regression Training, v. 6.0-35; http://caret.r-forge.r-project.org/) for model cross-validation (leave-one-out cross-validation to identify optimal GLMNet alpha and lambda tuning parameters).

Two models were used. Model A, the optimal classification model for ADEPT-asthma baseline clusters (Model A), based on the 8 clinical clustering variables (excluding PC20), was applied to classify U-BIOPRED subjects $(n=397$, including patients on OCS and smokers), limited only to those without missing data for these 8 clustering variables. PC20 was not included in the classification because this variable was not longitudinally assessed in ADEPT and was unavailable for most UBIOPRED participants. An alternate classification model, 
Model B, was built for longitudinal assessment of ADEPT asthma subjects (based on 3, 6, and 12 month data), excluding blood eosinophil counts (in addition to excluding PC20) because this variable was only measured at screening in ADEPT. To classify ADEPT subjects across the 3, 6 and 12 month visits and U-BIOPRED subjects into the determined clinical clusters based on the variables used in the original clustering of screening/baseline values for ADEPT-asthma subjects. Subjects were excluded from the classification analyses if they had missing data for at least one of the model variables at that time point.

The linear predictor coefficients for Model A and Model $B$ needed to calculate probabilities of classification to the ADEPT baseline clinical cluster groups are reported in Additional file 1: Table S3. The probability for classification of subject $i$ to outcome class $k=c$ (of $\mathrm{K}$ outcome classes) is determined by first calculating the set of linear predictors $f(\mathrm{k}, \mathrm{i})$ for subject $i$ at each outcome class $k: f(k, i)=\sum_{m=}$ ${ }_{0}^{M}\left(\beta m, k \cdot \boldsymbol{X}_{m, i}\right)$, where $\beta_{\mathrm{m}, \mathrm{k}}$ is the linear predictor function coefficient for predictor $m$ at outcome class $k$ (with the coefficient at predictor $m=0$ is the constant for the linear predictor function for class $k$ ) and $\boldsymbol{X}_{\mathrm{m}, \mathrm{i}}$ is the value of predictor $m$ for subject $i$.

The probability for classification of subject $i$ to outcome class $c\left[\operatorname{Pr}\left(Y_{i}=c\right)\right]$ is the ratio of the exponentiated linear predictor for outcome class $c$ over the sum of the exponentiated linear predictors across the $\mathrm{K}$ linear predictors for subject i: $\operatorname{Pr}\left(Y_{i}=c\right)=e^{f(c, i)} / \sum_{k=1}^{K} e^{f(k, i)}$. The outcome class with the highest probability for subject $i$ is the outcome class assigned to the subject.

An interactive calculator (file: ADEPT_clinical_cluster.Classification_calculator.Model_A.xlsx) allowing readers to input values for the 8 clustering variables, with the ADEPT clinical cluster assignment and classification probabilities automatically calculated for 'Model A'.

\section{Biologic sample acquisition and analysis Bronchoscopic sampling and transcriptomic analysis}

Endobronchial biopsies and epithelial brushings, taken at bifurcations of sub-segmental airways in the lower lobes, were immediately preserved in RNAlater solution and then maintained at $-70{ }^{\circ} \mathrm{C}[10,11]$. RNA was extracted using Qiagen miRNeasy kit (Qiagen; Germantown, MD) and amplified with NuGen ovation pico WTA kit (NuGen Technologies; San Carlos, CA). The cDNA was analyzed using the Affymetrix HG-U133 + PM microarray platform (Affymetrix, Santa Clara, CA). CEL files were normalized, assessed for quality control to exclude technical outliers (chip image analysis, Affymetrix GeneChip QC, RNA degradation analysis, distribution analysis, principal components analysis, and correlation analysis), and re-normalized using the robust multi-array (RMA) method. The $\log _{2}$-normalized data matrix was imported into OmicSoft ArrayStudio software (Cary, NC; www.omicsoft.com) for subsequent analysis. For ADEPT biopsies and U-BIOPRED brushings, batch effects from RNA processing sets were observed ( 2 sets for each sample type), with the batch effect adjusted in the data matrices using linear modeling of batch (as random factor) and cohort. A $\log _{2}$-intensity threshold of 5.5 for ADEPT biopsies and 4.75 for U-BIOPRED brushings was established as the limit of reliable quantification (LOD) based on the $90^{\text {th }}$ percentile signal of merged nonspecific probesets distribution in the array and by the inflection point of maximum variance with decreasing signal in a standard deviation vs. mean intensity plot across all probesets. Probesets with mean $\log _{2}$ intensity above this threshold in at least one of the 4 study cohorts were considered quantifiable and included in subsequent analyses (24033 and 21363 probesets for biopsies and brushings, respectively).

\section{Induced sputum (IS) sampling and analysis}

The sputum induction and processing protocols are published in detail elsewhere $[10,11,16]$. They differed in respect of duration of induction (maximum three 7min sessions of nebulization in ADEPT and four 5-min sessions in U-BIOPRED) and concentration of hypertonic saline (increasing concentrations of 3, 4, and 5\% for ADEPT and 0.9 to $4.5 \%$ for U-BIOPRED).

For both cohorts, mucoid portions of sputum were selected and treated with dithiothreitol for this study in all participants [17]. Sputum supernatant and cytospin slides for differential cell counts were prepared by standard methods. Standard differential staining and counting was performed centrally. For U-BIOPRED, assessments of a maximum of 400 inflammatory cells on Diff-Quick stained cytospins were performed centrally with the outcome of the cytospin analysis. Sample viability $\geq 50 \%$ and a threshold of $\leq 40 \%$ squamous cells was the default for samples being made available for analysis. For both studies, only samples with squamous cell content $\leq 30 \%$ from cytospin differential counts were included in the analyses. For ADEPT, a significant proportion of subjects had only a screening or only a baseline sample available that passed quality control standards. Therefore, the mean (differential cell counts) or geometric mean (analyte, gene expression measurements) of screening and baseline measurements was used for subsequent analyses.

\section{Serum sampling and analysis}

Serum was collected using standard Serum Separation Tubes (SST), frozen within $30 \mathrm{~min}$ and subsequently used for quantification of 1129 serum analytes applying the SomaScan v3 platform. Results for serum total immunoglobulin E (IgE) are presented from this panel, defining high IgE levels as those above the $95^{\text {th }}$ percentile of the $\mathrm{HV}$ distribution. In previous evaluations of the 
platform in asthmatics and HV, IgE measurements highly correlated (Pearson's correlation coefficient $r>0.9$ ) with those obtained from standard ELISA-based assays (data not shown).

\section{Airway type-2 inflammation high and low phenotype} For ADEPT, airway Th2-high status was defined on the basis of biopsy CCL26 gene expression (from microarray data) beyond the healthy control distribution, which also coincided with the limit of reliable quantification (LOQ) for the CCL26 probeset. Periostin (POSTN) gene expression (from microarray data) beyond the healthy control distribution was evaluated as an additional indicator of T2-high status. Endobronchial brushings samples from ADEPT were limited in sample size and therefore not applicable for assigning airway Th2-high status for subjects across the clinical clusters.

For U-BIOPRED, endobronchial brushings were selected as the airway sample type to evaluate Th2-high status because this was the sample type with the largest sample size and most overlap with other sample types (biopsies, sputum). Because CCL26 expression was below LOQ for most subjects across the study cohorts in U-BIOPRED biopsies and was also below LOQ in the brushings, an alternative Th2-activity indicator was selected, namely our own IL-13 ex vivo stimulation gene signature (IL-13 IVS) in endobronchial epithelial air-liquid interface cultures. The signature (genes in signature listed in Additional file 1: Table S4) was defined by selecting genes commonly induced by IL-13 stimulation across 3 independent sets of experiments (data not shown). Enrichment was evaluated on a per-subject basis using the R-Bioconductor package Gene Set Variation Analysis (GSVA, v 1.14.1) [18]. Subjects with enrichment scores (ES) beyond the $95^{\text {th }}$ percentile of healthy control distribution were classified as airway T2-high.

\section{Phadiatop ${ }^{\circledR}$ testing}

The ImmunoCAP Phadiatop test (USA or Europe regional test, depending on the patients' respective regions) (http:// www.phadia.com/en-GB/5/Products/ImmunoCAP-Assays /1/) [19] was used to determine the atopic status of patients. ImmunoCAP Phadiatop is a blood test in which results are expressed as positive or negative. A positive Phadiatop ${ }^{\circ}$ result indicates that the patient is atopic. A negative result indicates that the patient is non-atopic, i.e. not sensitized to inhalant allergens.

\section{Results}

\section{The ADEPT clinical phenotypes}

The ADEPT asthmatics $(n=156)$ were partitioned into clusters, i.e. clinical phenotypes, based on baseline data of the 9 clustering variables. Partitioning the asthma population into four clusters was determined to be optimal based on maximizing between-cluster Silhouette metric $\mathrm{Fc}(\mathrm{U})$ and minimizing within-cluster distance metric $\mathrm{Dc}(\mathrm{U})$. The $\mathrm{Fc}(\mathrm{U}) / \mathrm{Dc}(\mathrm{U})$ values for options with $2,3,4$, and 5 clusters were: $0.742 / 0.110,0.768 / 0.080$, $0.786 / 0.079$ (optimal), and $0.772 / 0.082$, respectively. $>75 \%$ of participants in each cluster had $>80 \%$ probability for their assigned phenotype, and only one subject per phenotype had $<50 \%$ probability of 'belonging' to their phenotype (Fig. 2). The clinical and biomarker characteristics of the 4 ADEPT phenotypes (Clusters A1 to A4) are shown in Fig. 3 and Table 1 and graphically summarized in Fig. 4.

Phenotype A1 ('mild, normal lung function, early onset, low inflammation') comprised mostly mild asthmatics (mean ACQ-7: 0.5) and the vast majority (93\%) were not currently on ICS. This was the phenotype with the lowest mean age-of-onset (15 yrs), BDR and AHR, and preserved lung function that was not different from healthy participants. Their inflammatory burden was low, based on mean FENO levels, the second lowest blood and sputum eosinophil counts, and the lowest sputum neutrophil counts.

Phenotype A2 ('moderate, mild reversible obstruction, hyper-responsive, highly atopic, eosinophilic') contained mostly moderate $(52 \%)$ and to a lesser degree severe asthmatics (32\%), as defined at the time of recruitment, characterized by mild, reversible airflow obstruction, and moderate asthma activity (mean ACQ-7: 1.1). However, this phenotype had the most hyper-responsive asthmatics, as measured by methacholine $\mathrm{PC}_{20}$, with the highest degree of eosinophilic inflammation (based on FENO and blood and sputum eosinophils), and they were the most atopic (based on total and specific serum IgE (Phadiatop $\left.{ }^{\circ}\right)$.

Phenotype A3 ('mixed severity, mild reversible obstruction, non-eosinophilic, neutrophilic') consisted of a mix of asthmatics with mild (27\%), moderate (43\%), and severe (31\%) disease. The majority were reasonably wellcontrolled and had mild airflow obstruction and AHR, less BDR (approximately $1 / 3$ participants irreversible) and reduced FVC. Cluster A3 had the lowest FENO, the least eosinophilic inflammation, the lowest rate of atopy, but the highest sputum neutrophils.

Phenotype A4 ('severe uncontrolled, severe reversible obstruction, mixed granulocytic) asthmatics were derived predominantly from the severe $(63 \%)$ and to a lesser extent moderate (26\%) asthmatics. They were generally the most severe and uncontrolled, with the greatest airflow obstruction, highest BDR, and high degree of AHR and were characterized by prominent mixed eosinophilic/neutrophilic inflammation. Although this phenotype had similar high proportions of serum IgE-high participants as Phenotype A2, the rate of atopy (Phadiatop ${ }^{\circ}$ test, see Methods) was lower, similar to Cluster A3. 


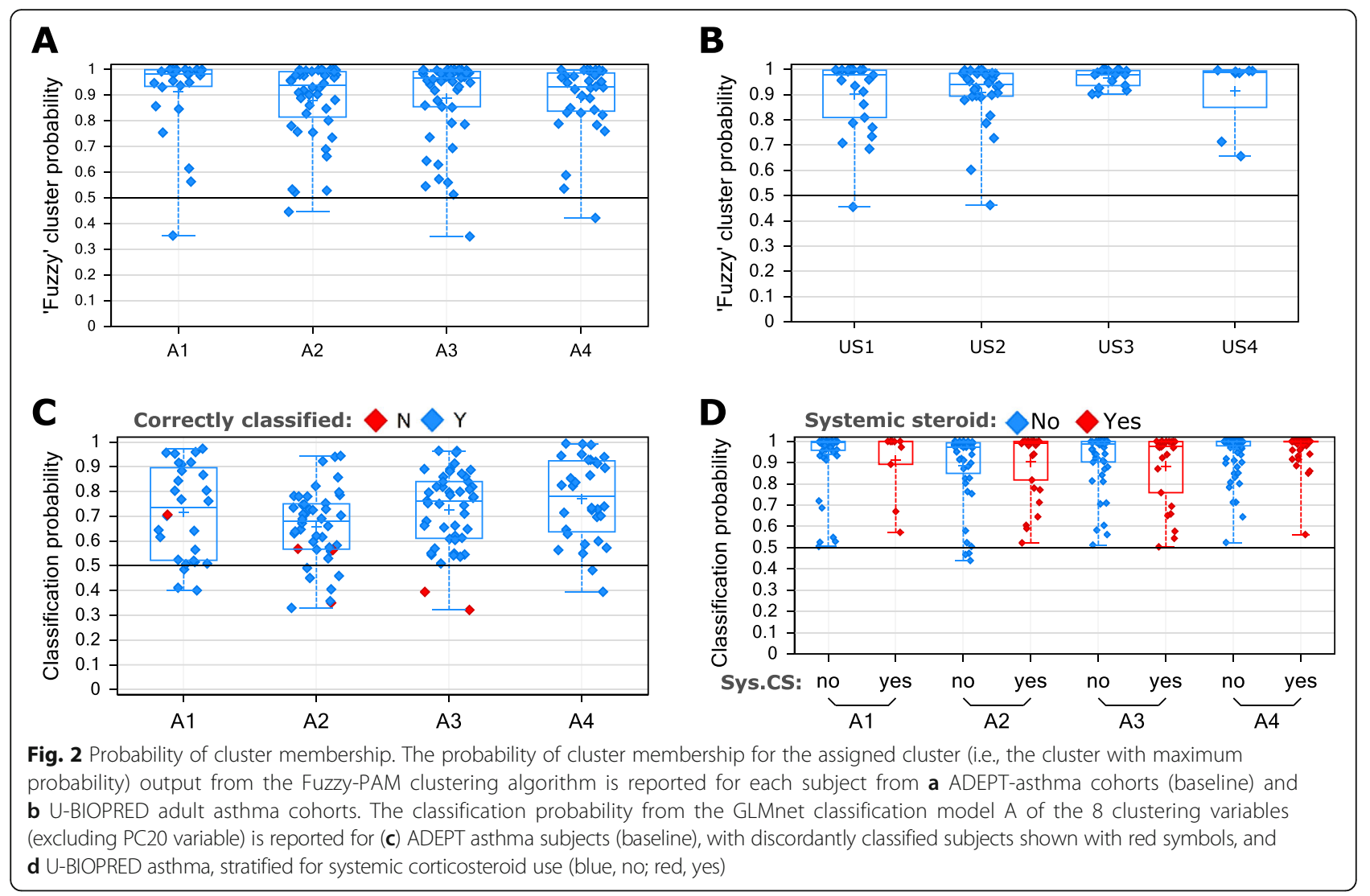

All 9 clustering variables significantly varied across the 4 ADEPT phenotypes as shown in Fig. 3 and Table 1, which report additional clinical, biomarker, and demographic variables. When restricting analysis to moderate and severe asthma, the 9 clustering variables also significantly varied across Phenotypes A2, A3, and A4 (Table 2).

\section{The ADEPT phenotypes resist perturbation}

Sensitivity analyses were performed to confirm robustness of the clusters to perturbations of removing groups of subjects. In the first sensitivity analysis, only 1 of 152 subjects changed cluster assignment after excluding the 'medoid' subject defining each cluster group. Next, only 4 of 104 subjects from original clusters 2, 3, and 4 changed cluster assignment after the mild asthma cohort was excluded, with 3 clusters selected for analysis. Similarly, when the moderate asthma cohort was excluded, only 4 of 101 subjects changed cluster assignment. Only when the severe cohort was excluded was there a marked deterioration in cluster formation, with 37 of 105 changing cluster assignment, specifically with Group 4 not well-forming in this scenario. This was not unexpected given that the original Group 4 consisted mostly of severe asthma subjects.
The ADEPT phenotypes are stable longitudinally

Applying "Model B" (excluding blood eosinophils and $\mathrm{PC}_{20}$ ) to classify ADEPT participants for the baseline, 3, 6 and 12-month visits, stratified by the original baseline clustering assignment, 24 of 147 participants (16\%) had a discordant baseline classification relative to their originally assigned baseline phenotype (i.e., 16\% error rate in classification performance). By comparison using Model A (including blood eosinophils but not $\mathrm{PC}_{20}$ ) to classify baseline samples, discordance was reduced to a $4 \%$ error in classification to the originally assigned phenotype (Fig. 2). Fig. 5 displays the frequency of subjects that maintain or change phenotype classification across the longitudinal visits. In general, the majority of participants had stable phenotype assignments throughout the 12-month duration of the study. The Pearson's Contingency Coefficients representing the within-subject consistency of classified phenotype across visits were $0.81,0.74,0.76$, and 0.78 for actual baseline phenotypes A1, A2, A3, and A4, respectively. The overall discordance for classified vs. originally assigned phenotypes was $31 \%, 31 \%$, and $40 \%$ for the 3,6 , and 12 month visits (compared to the baseline discordance error rate of 16\%) (Fig. 5). Original baseline Phenotype A4 showed the most changes at follow-up visits, with $39 \%, 40 \%$, and $40 \%$ of participants with discordant classifications at the 


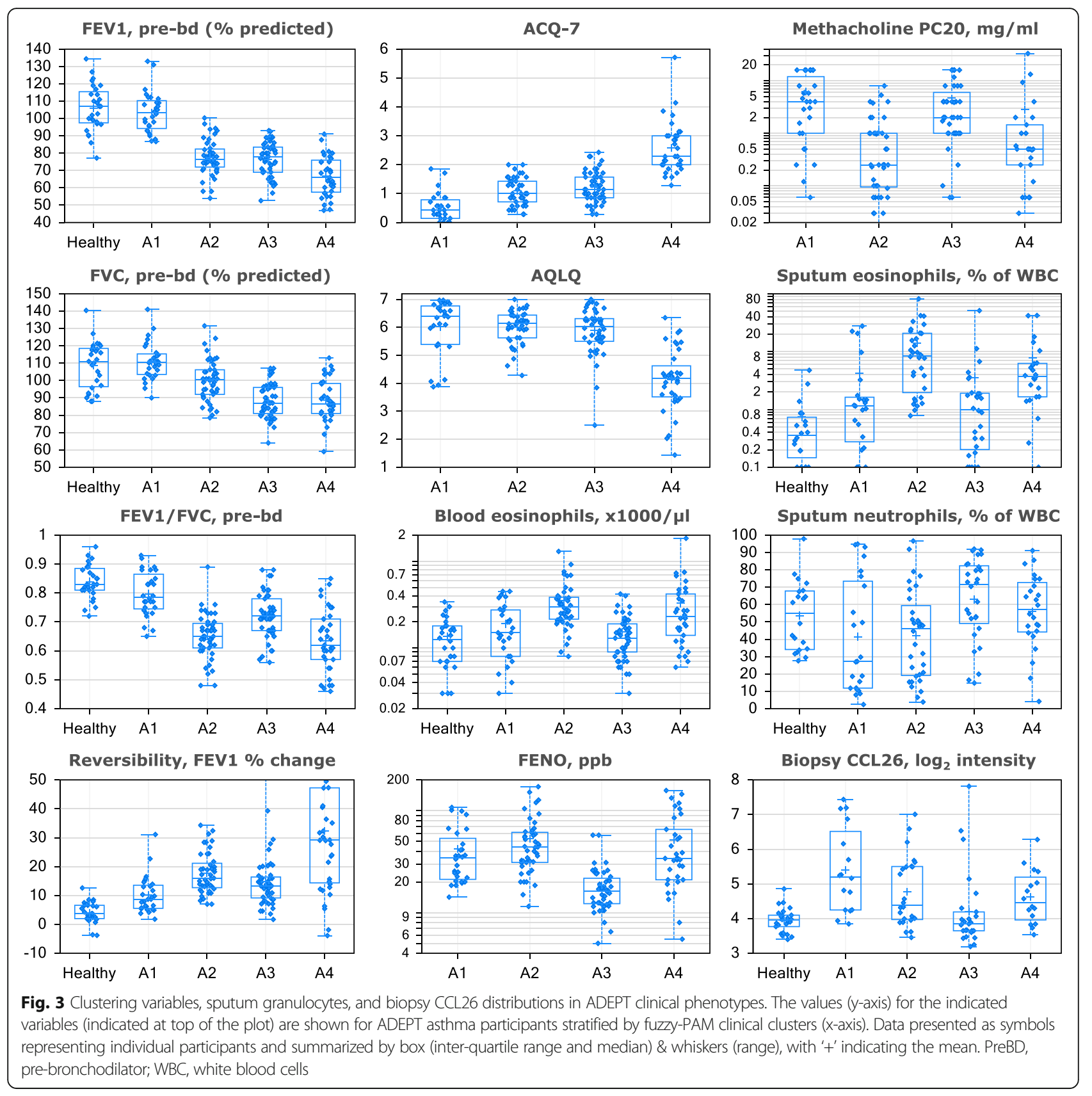

3,6 , and 12 month visits, respectively. Additional file 1 : Figure S1 shows how subjects changed cluster classifications over time for subjects having discordant classifications for at least one-time point compared to the original, baseline clustering assignment. Case-reports for associated changes in clinical and biomarker variables are described in Additional file 1: Figure S2 for subjects having discordant classifications compared to baseline (one random subject per baseline cluster selected for presentation) and in Additional file 1: Figure S3 for subjects having concordant classifications across visits compared to baseline assignment (one random subject per baseline cluster selection for presentation).

\section{The ADEPT matched U-BIOPRED sub-population also optimally partitions into 4 phenotypes}

For the U-BIOPRED cohort [11], 82 out of 397 participants were selected for clustering based on similar clinical inclusion criteria as ADEPT participants excluding U-BIOPRED participants on OCS as well as smokers. When compared to ADEPT moderate-severe asthmatics, the restricted UBIOPRED set had slightly lower $\mathrm{FEV}_{1}$ (mean \pm standard 
Table 1 Clinical, biomarker, and demographic characteristics of ADEPT clusters at baseline

\begin{tabular}{|c|c|c|c|c|c|c|}
\hline Variable $^{a}$ & $\begin{array}{l}\text { Healthy } \\
(n=31)\end{array}$ & $\begin{array}{l}\text { Cluster A1 } \\
(n=28)\end{array}$ & $\begin{array}{l}\text { Cluster A2 } \\
(n=44)\end{array}$ & $\begin{array}{l}\text { Cluster A3 } \\
(n=49)\end{array}$ & $\begin{array}{l}\text { Cluster A4 } \\
(n=35)\end{array}$ & $P$-value \\
\hline \multicolumn{7}{|l|}{ Mean $[$ Geo.Mean] \pm SD } \\
\hline FEV1, pre-bd, \% predicted & $106.1 \pm 13.5$ & $103.5 \pm 11.8$ & $77.4 \pm 10.2$ & $76.5 \pm 9.8$ & $66.8 \pm 11.5$ & $<10^{-6}$ \\
\hline FVC, pre-bd, \% predicted & $108.8 \pm 13.6$ & $111.1 \pm 10.8$ & $100.0 \pm 12.0$ & $88.2 \pm 9.8$ & $88.7 \pm 12.0$ & $<10^{-6}$ \\
\hline FEV1/FVC, pre-bd & $0.84 \pm 0.06$ & $0.80 \pm 0.08$ & $0.65 \pm 0.08$ & $0.72 \pm 0.08$ & $0.64 \pm 0.11$ & $<10^{-6}$ \\
\hline bd reversibility, \% change FEV1 & $4.1 \pm 3.6$ & $10.2 \pm 6.3$ & $17.6 \pm 7.0$ & $14.8 \pm 9.6$ & $32.2 \pm 21.9$ & $<10^{-6}$ \\
\hline ACQ7 & na & $0.5 \pm 0.5$ & $1.1 \pm 0.5$ & $1.2 \pm 0.5$ & $2.6 \pm 0.9$ & $<10^{-6}$ \\
\hline AQLQ & na & $6.0 \pm 1.0$ & $6.0 \pm 0.6$ & $5.9 \pm 0.8$ & $4.1 \pm 1.1$ & $<10^{-6}$ \\
\hline FENO, ppb [Geo.Mean] & na & $35.2+28.1 /-15.6$ & $43.8+36.7 /-20.0$ & $16.4+9.7 /-6.1$ & $37.7+47.8 /-21.1$ & $<10^{-6}$ \\
\hline $\begin{array}{l}\text { Blood eosinophils, } \times 1000 / u l \\
\text { [Geo.Mean] }\end{array}$ & $0.112+0.106 /-0.054$ & $0.150+0.168 /-0.079$ & $0.299+0.234 /-0.131$ & $0.131+0.100 /-0.057$ & $0.237+0.286 /-0.130$ & $<10^{-6}$ \\
\hline PC20, mg/ml [Geo.Mean] & na & $2.8+11.3 /-2.2$ & $0.3+1.4 /-0.3$ & $2.2+7.0 /-1.7$ & $0.6+2.8 /-0.5$ & $<10^{-6}$ \\
\hline $\begin{array}{l}\text { Blood neutrophils, ×1000/ul } \\
\text { [Geo.Mean] }\end{array}$ & $3.47+1.50 /-1.05$ & $3.26+1.03 /-0.78$ & $3.83+1.56 /-1.11$ & $3.93+1.65 /-1.16$ & $3.73+1.29 /-0.96$ & 0.0999 \\
\hline $\begin{array}{l}\text { Blood WBC, } \times 1000 / u l \\
\text { [Geo.Mean] }\end{array}$ & $6.01+1.72 /-1.34$ & $6.01+1.43 /-1.16$ & $6.42+2.04 /-1.55$ & $6.32+2.12 /-1.59$ & $6.49+1.38 /-1.14$ & 0.6676 \\
\hline Sputum neutrophils, $\%$ of WBC ${ }^{b}$ & $53.6 \pm 20.1$ & $41.4 \pm 33.0$ & $42.2 \pm 25.8$ & $63.1 \pm 23.8$ & $55.9 \pm 21.7$ & 0.0053 \\
\hline $\begin{array}{l}\text { Sputum eosinophils, \% of WBC } \\
\text { [Geo.Mean] }]^{\mathrm{b}}\end{array}$ & $0.4+0.8 /-0.3$ & $1.0+4.7 /-0.8$ & $7.1+18.7 /-5.1$ & $0.9+3.5 /-0.7$ & $3.5+10.5 /-2.6$ & $<10^{-6}$ \\
\hline Serum IgE, RFU [Geo.Mean] & $1.0+2.1 /-0.7$ & $7.6+29.5 /-6.0$ & $12.6+27.5 /-8.6$ & $7.1+20.3 /-5.3$ & $14.4+33.2 /-10.0$ & 0.0388 \\
\hline Age, years & $31.5 \pm 9.1$ & $32.5 \pm 14.3$ & $42.4 \pm 12.0$ & $43.0 \pm 11.8$ & $46.8 \pm 13.3$ & 0.0002 \\
\hline Age of onset, years & na & $15.4 \pm 13.9$ & $21.0 \pm 14.0$ & $22.7 \pm 15.2$ & $23.7 \pm 18.6$ & 0.1537 \\
\hline Body Mass Index (kg/m²) & $24.7 \pm 3.3$ & $25.2 \pm 3.2$ & $25.7 \pm 3.6$ & $26.9 \pm 3.7$ & $26.6 \pm 4.1$ & 0.1622 \\
\hline \multicolumn{7}{|l|}{ Percent of clinical cluster } \\
\hline Mild, no ICS (\%) & na & $93 \%$ & $16 \%$ & $27 \%$ & $11 \%$ & \\
\hline Moderate, low-medium ICS (\%) & na & $7 \%$ & $52 \%$ & $43 \%$ & $26 \%$ & $<10^{-6}$ \\
\hline Severe, high ICS (\%) & na & $0 \%$ & $32 \%$ & $31 \%$ & $63 \%$ & \\
\hline Male (\%) & $65 \%$ & $46 \%$ & $48 \%$ & $43 \%$ & $43 \%$ & 0.9500 \\
\hline Atopic, Phadiatop positive (\%) & $0 \%$ & $79 \%$ & $91 \%$ & $69 \%$ & $71 \%$ & 0.0670 \\
\hline $\begin{array}{l}\text { Serum IgE-high, }>95 \text { th \%ile of } \\
\text { controls (\%) }\end{array}$ & $0 \%$ & $71 \%$ & $86 \%$ & $69 \%$ & $83 \%$ & 0.1692 \\
\hline $\begin{array}{l}\text { Sputum Pauci (Eos }<\% 3 \text {, } \\
\text { PMN < 60\%) }(\%)^{b}\end{array}$ & $45 \%$ & $48 \%$ & $16 \%$ & $36 \%$ & $21 \%$ & \\
\hline $\begin{array}{l}\text { Sputum Neutr. (Eos }<3 \\
\text { PMN }>=60 \%)(\%)^{b}\end{array}$ & $50 \%$ & $30 \%$ & $13 \%$ & $43 \%$ & $17 \%$ & 0.0012 \\
\hline $\begin{array}{l}\text { Sputum Mix (Eos } \geq 3 \% \text {, } \\
\text { PMN }>=60 \%)(\%)^{b}\end{array}$ & $0 \%$ & $0 \%$ & $13 \%$ & $14 \%$ & $29 \%$ & \\
\hline $\begin{array}{l}\text { Sputum Eos. (Eos } \geq 3 \% \text {, } \\
\text { PMN }<60 \%)(\%)^{b}\end{array}$ & $5 \%$ & $22 \%$ & $59 \%$ & $7 \%$ & $33 \%$ & \\
\hline FENO $\geq 35$ ppb (\%) & na & $50 \%$ & $61 \%$ & $4 \%$ & $44 \%$ & $<10^{-6}$ \\
\hline Blood eosinophils $\geq 300 /$ ul (\%) & $6 \%$ & $21 \%$ & $50 \%$ & $6 \%$ & $34 \%$ & $2.9 \times 10^{-5}$ \\
\hline $\begin{array}{l}\text { FENO } \geq 35 \text { ppb OR Blood eos. } \\
\geq 300 / \text { ul (\%) }\end{array}$ & na & $57 \%$ & $84 \%$ & $10 \%$ & $57 \%$ & $<10^{-6}$ \\
\hline Biopsy CCL26-high (\%) ${ }^{c}$ & $0 \%$ & $56 \%$ & $42 \%$ & $16 \%$ & $31 \%$ & 0.0515 \\
\hline
\end{tabular}

${ }^{a}$ Summary statistics (mean, or geometric mean where indicated, and standard deviation; or percent of clinical cluster) and $p$-values from F-test (for mean/geomtric mean statistics) or $\mathrm{x}$-square statistics for associations among the clinical clusters (not included healthy control cohort) are presented. The 9 clinical and clinical biomarker variables used in the clustering are presented first, followed by additional clinical, biomarker and demographic variable groupings

${ }^{b}$ For sputum differentials, $n=20,23,32,38$, and 24 for Healthy cohort and Clusters 1, 2, 3, and 4, respectively

${ }^{C}$ For endobronchial biopsy gene expression variables (from Affymetrix HG-U133 + PM array signal intensities), $n=25,27,28,33$, and 18 for Healthy cohort and Clusters 1, 2, 3, and 4, respectively

Significant $p$ values are captured in bold 


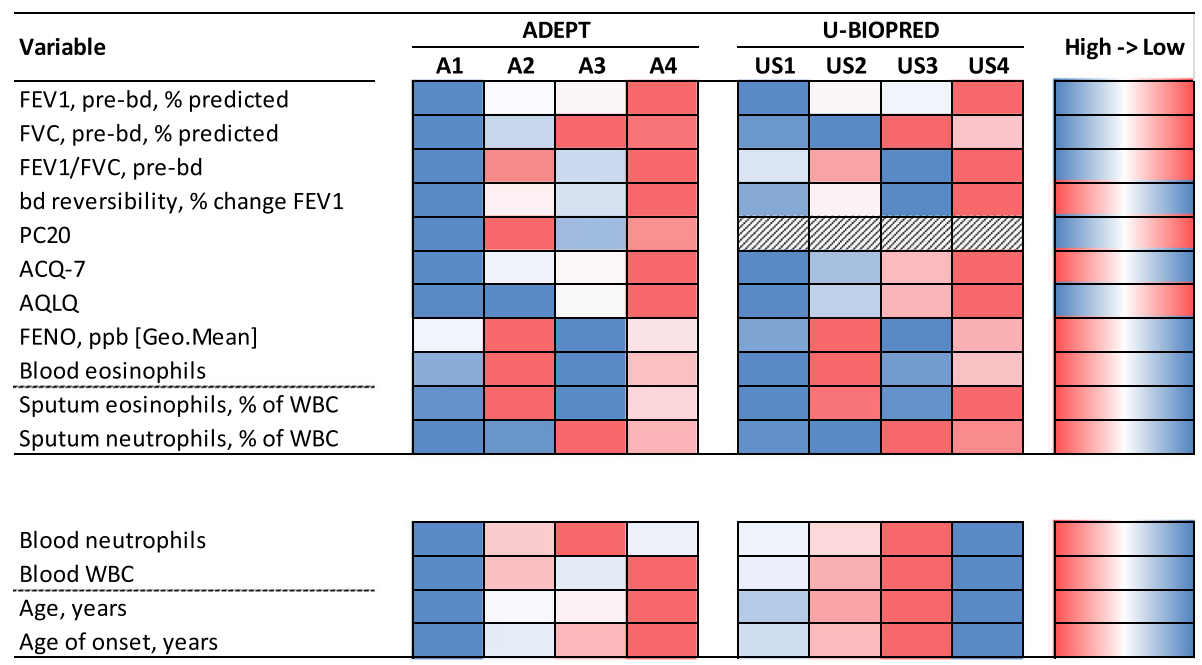

Fig. 4 Mean values of clustering and sputum granulocyte variables among clinical clusters. Relative mean values of the indicated variables are schematically represented for each clinical cluster from 'best' (blue) to 'worst' (red) values among clusters within the indicated study (coloring for high-to-low values of variable indicated in right-most column)

deviations of $71.9 \pm 10.7$ vs. $68.1 \pm 9.8, p=0.011)$, FVC (91.6 \pm 11.6 vs. $86.5 \pm 14.1, p=0.007)$, and AQLQ (5.4 \pm 1.2 vs. $5.0 \pm 1.2, p=0.018)$ and higher ACQ-7 (1.6 \pm 0.9 vs. $2.3 \pm 1.2, p=0.000045)$. This also resulted in an optimum of 4 phenotypes, based on $\mathrm{Fc}(\mathrm{U}) / \mathrm{Dc}(\mathrm{U})$ partition metrics of $0.776 / 0.060,0.810 / 0.054,0.837 / 0.049$ (optimal), and 0.800/0.080, for options with $2,3,4$, and 5 clusters, respectively. The U-BIOPRED phenotypes, US (U-BIOPRED subset)- 1 to US4, shared similar characteristics as A1, A2, A3 and A4, respectively. More than $75 \%$ of participants in each cluster had $>81 \%$ probability for their assigned phenotype, and only 2 participants (in Phenotype US1 and US2) had $<50 \%$ probability of belonging to their phenotype (Fig. 2). All clustering variables were significantly associated across the 4 phenotypes. Figure 6 and Table 3 show clustering, clinical, biomarker, and demographic variables for the US1 to US4 clusters.

Phenotype US1 ('mild, good lung function, early onset, low inflammation') was the least symptomatic, with modest reversible obstruction and good asthma control. Eosinophilic inflammation was minimal (see Table 3: FENO, blood eosinophils). US1 was most similar to A1, albeit with a greater BDR and lower FENO levels, the latter perhaps due to ICS treatment in US1 but not A1 participants. US1 was generally more severe than A1.

Phenotype US2 ('moderate, hyper-responsive, eosinophilic') in general is characterized by reversible obstruction, uncontrolled asthma, normal FVC, and predominantly eosinophilic inflammation, with $88 \%$ of participants being FENO-high $(\geq 35 \mathrm{ppb})$ or blood eosinophil-high $(\geq 300 / \mu \mathrm{L})$. For those with induced sputum, most $(69 \%)$ had high sputum eosinophils $(\geq 3 \%$ of leukocytes) and few (12\%) had high sputum neutrophils ( $\geq 60 \%$ of leukocytes). Phenotype US2 was largely homologous to phenotype A2.

Phenotype US3 ('mixed severity, mild reversible obstruction, non-eosinophilic, neutrophilic') in general had moderately reduced FEV1, reduced mean FVC $(72.9 \pm 11.5 \% \%$ predicted), poor asthma control, and low-to-modest BDR. Phenotype US3 was neutrophilic and non-eosinophilic, with only $41 \%$ of participants being FENO-high or blood eosinophil-high, compared to $21 \%$ observed in the healthy control group. The majority of participants (63\%) were sputum neutrophil-high, and few (12.5\%) were sputum eosinophil-high. Phenotype US3 was, therefore, largely homologous to Phenotype A3.

Phenotype US4 ('severe uncontrolled, severe reversible obstruction, mixed granulocytic') in general was characterized by marked airflow obstruction, high BDR with reduced FVC, and very poor asthma control. These participants were eosinophilic, with $88 \%$ of the phenotype being FENO-high or blood eosinophil-high and $75 \%$ having elevated sputum eosinophils, but $50 \%$ also having high sputum neutrophils. Phenotype US4 was homologous to Phenotype A4.

Plethysmography data were available in the UBIOPRED study (Additional file 1: Table S5). Participants in Phenotype US4 had relatively normal total lung capacity but elevated residual volumes $(67 \%$ higher than HV), and $24-40 \%$ higher than Phenotypes US1, US2, and US3. These results indirectly suggest that the reduced FVC (mean $81 \%$ predicted) was a consequence of air trapping for Phenotype US4. 
Table 2 Clinical, biomarker, and demographic associations among the ADEPT moderate-severe asthma subjects in baseline clinical cluster groups

\begin{tabular}{|c|c|c|c|c|}
\hline Variable $^{a}$ & Phenotype A2 $(n=37)$ & Phenotype A3 $(n=36)$ & Phenotype A4 $(n=31)$ & $P$-value \\
\hline \multicolumn{5}{|l|}{ Mean [Geo.Mean] $\pm S D$} \\
\hline FEV1, pre-bd, \% predicted & $74.6 \pm 8.2$ & $73.8 \pm 9.8$ & $65.5 \pm 11.5$ & $3.0 \times 10^{-4}$ \\
\hline FVC, pre-bd, \% predicted & $97.4 \pm 9.8$ & $87.4 \pm 10.2$ & $88.6 \pm 12.2$ & $2.0 \times 10^{-4}$ \\
\hline FEV1/FVC, pre-bd & $0.64 \pm 0.08$ & $0.70 \pm 0.07$ & $0.62 \pm 0.10$ & $9.0 \times 10^{-4}$ \\
\hline bd reversibility, \% change FEV1 & $17.3 \pm 6.6$ & $16.5 \pm 10.5$ & $32.0 \pm 21.5$ & $1.1 \times 10^{-5}$ \\
\hline ACQ-7 & $1.1 \pm 0.4$ & $1.3 \pm 0.6$ & $2.6 \pm 0.9$ & $<10^{-6}$ \\
\hline AQLQ & $6.0 \pm 0.6$ & $5.8 \pm 0.9$ & $4.1 \pm 1.2$ & $<10^{-6}$ \\
\hline FENO, ppb [Geo.Mean] & $42.0+35.9 /-20.9$ & $15.6+8.8 /-7.9$ & $37.1+47.9 /-17.8$ & $<10^{-6}$ \\
\hline Blood eosinophils, ×1000/ul [Geo.Mean] & $0.317+0.244 /-0.134$ & $0.124+0.094 /-0.037$ & $0.235+0.285 /-0.151$ & $<10^{-6}$ \\
\hline PC20, mg/ml [Geo.Mean] & $0.5+1.8 /-0.5$ & $2.2+8.8 /-2.2$ & $0.4+1.4 /-0.4$ & $1.0 \times 10^{-4}$ \\
\hline Blood neutrophils, ×1000/ul [Geo.Mean] & $3.80+1.64 /-3.80$ & $3.95+1.78 /-3.95$ & $3.67+1.33 /-3.67$ & 0.6916 \\
\hline Blood WBC, ×1000/ul [Geo.Mean] & $6.34+2.10 /-0.35$ & $6.35+2.28 /-0.29$ & $6.44+1.39 /-0.44$ & 0.9687 \\
\hline Sputum neutrophils, \% of WBC ${ }^{b}$ & $43.1 \pm 26.6$ & $61.6 \pm 25.1$ & $56.2 \pm 22.1$ & 0.0239 \\
\hline Sputum eosinophils, \% of WBC [Geo.Mean] $]^{\mathrm{b}}$ & $7.8+20.7 /-0.6$ & $0.7+2.7 / 0.0$ & $3.1+8.5 /-0.2$ & $<10^{-6}$ \\
\hline Age, years & $43.0 \pm 12.3$ & $45.6 \pm 10.7$ & $47.9 \pm 12.3$ & 0.2340 \\
\hline Age of onset, years & $20.9 \pm 14.2$ & $23.4 \pm 15.5$ & $26.1 \pm 18.3$ & 0.4040 \\
\hline Body Mass Index (kg/m²) & $25.7 \pm 3.6$ & $27.3 \pm 3.7$ & $26.8 \pm 4.2$ & 0.2308 \\
\hline \multicolumn{5}{|l|}{ Percent of clinical cluster group } \\
\hline Mild, no ICS (\%) & na & na & na & \\
\hline Moderate, low-medium ICS (\%) & $62 \%$ & $58 \%$ & $29 \%$ & 0.0135 \\
\hline Severe, high ICS (\%) & $38 \%$ & $42 \%$ & $71 \%$ & \\
\hline Male (\%) & $49 \%$ & $44 \%$ & $48 \%$ & 0.9240 \\
\hline Atopic, Phadiatop positive (\%) & $92 \%$ & $72 \%$ & $71 \%$ & 0.0530 \\
\hline Sputum Pauci (Eos < \%3, PMN < 60\%) $(\%)^{\mathrm{b}}$ & $10 \%$ & $38 \%$ & $22 \%$ & \\
\hline Sputum Neutr. (Eos < 3, PMN > =60\%) $(\%)^{b}$ & $14 \%$ & $46 \%$ & $17 \%$ & 0.0003 \\
\hline Sputum Mix (Eos $>=3 \%$, PMN $>=60 \%)(\%)^{b}$ & $14 \%$ & $8 \%$ & $30 \%$ & \\
\hline Sputum Eos. (Eos > =3\%, PMN <60\%) $(\%)^{\mathrm{b}}$ & $62 \%$ & $8 \%$ & $30 \%$ & \\
\hline FENO $\geq 35$ ppb (\%) & $59 \%$ & $3 \%$ & $43 \%$ & $1.8 \times 10^{-6}$ \\
\hline Blood eosinophils $\geq 300 /$ lul (\%) & $54 \%$ & $6 \%$ & $32 \%$ & $4.1 \times 10^{-5}$ \\
\hline FENO $\geq 35$ ppb OR Blood eos. $\geq 300 /$ ul (\%) & $81 \%$ & $8 \%$ & $55 \%$ & $<10^{-6}$ \\
\hline Biopsy CCL26-high (\%) & $33 \%$ & $11 \%$ & $29 \%$ & 0.2179 \\
\hline
\end{tabular}

${ }^{a}$ Summary statistics (mean, or geometric mean where indicated, and standard deviation; or percent of clinical cluster group) and $p$-values from F-test (for mean/ geometric mean statistics) or chisquare statistics for associations among the ADEPT clinical cluster groups, restricted to moderate-severe asthma subjects and excluding Group 1, are presented. The 9 clinical and clinical biomarker variables used in the clustering are presented first, followed by additional clinical, biomarker and demographic variable groupings

${ }^{\mathrm{b}}$ For sputum differentials, $n=29,24$, and 23 for Groups 2, 3, and 4, respectively

${ }^{c}$ For endobronchial biopsy gene expression variables (from Affymetrix HG-U133 + PM array signal intensities), $n=21,20$, and 14 for Groups 2,3 , and 4, respectively Significant $p$ values are captured in bold

The U-BIOPRED study included several patientreported outcome (PRO) questionnaires not included in the ADEPT study: Epworth sleepiness scale, Hospital Anxiety and Depression Scale (HADS), Medication Adherence Report Scale (MARS), Sino-Nasal Outcome Test (SNOT), (Additional file 1: Table S5). Phenotypes US1, US3, and US4 subjects had higher scores for Epworth sleepiness scale compared to healthy participants $(p<0.05)$. Except for phenotype US1, HADS scores were elevated in each cluster compared to healthy participants $(p<0.05)$. All 4 clusters had significantly higher SNOT scores $\left(p<7 \times 10^{-5}\right)$, particularly for clusters US3 and US4. Importantly, all 4 U-BIOPRED phenotypes had similar scores on the MARS questionnaire, suggesting that clinical and biomarker differences between the clusters is not largely driven by differential adherence to medication usage. 

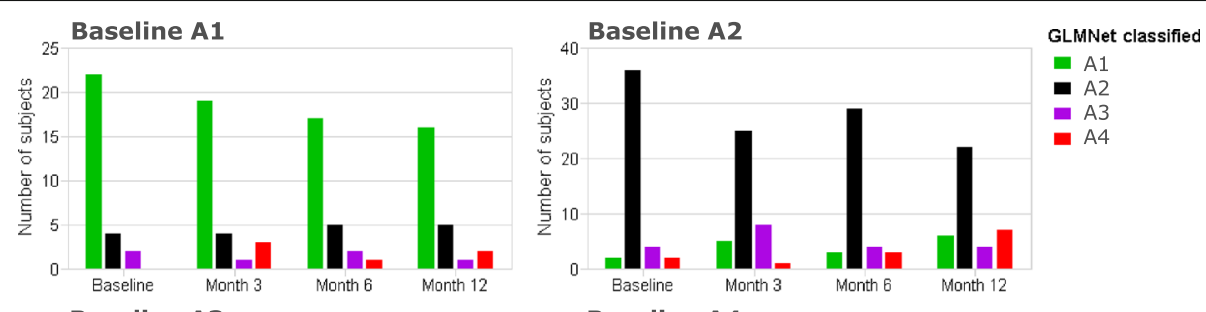

Baseline A3

Baseline A4
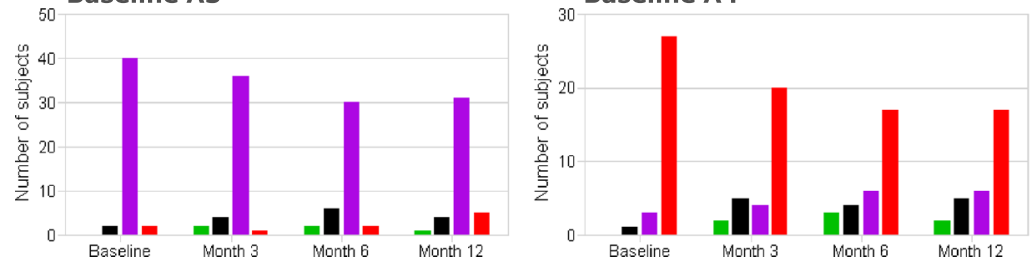

Fig. 5 Longitudinal evaluation of ADEPT-asthma clinical phenotype classification. GLMnet-classification model of ADEPT-asthma baseline clinical phenotypes (7 clustering variables, excluding PC20 and blood eosinophils; Model B) was applied to classify the ADEPT-asthma participants based on data from the baseline and 3, 6, and 12 month follow-up visits. Each panel presents ADEPT asthma participants assigned to the indicated clinical phenotypes from the baseline clustering analysis, reporting the phenotype to which they are classified at the indicated follow-up visits

\section{Classification of U-BIOPRED participants to the ADEPT baseline phenotypes}

As an alternate strategy to assess how well the ADEPT clinical cluster structure fits to an independent study population, GLMnet-classification model of ADEPTasthma baseline clinical clusters (8 clustering variables, excluding PC20; Model A) was applied to classify the subset of 397 participants from the U-BIOPRED asthma subjects (not restricted to ADEPT inclusion criteria and for whom all clustering clinical data were available). The classification probabilities for the classified ADEPT clusters are reported in Fig. 2 for both ADEPT (panel c, using baseline data to demonstrate model performance) and UBIOPRED (panel D), with stratification by systemic steroid use for U-BIOPRED. For ADEPT-asthma, the classification concordance to actual clusters was excellent at $96 \%$ overall for all clusters. When applying the classification model to U-BIOPRED, the probability distributions were similar to that in ADEPT. Remarkably, the maintenance OCS group, not represented in ADEPT, also fitted well into ADEPT phenotype classification structure, with most participants having $>80 \%$ probability for their classified cluster. Importantly, the relative distributions of the 8 clustering variables were similar across the 4 classified ADEPT clinical phenotypes for U-BIOPRED participants (Fig. 7) compared to ADEPT asthmatic participants (Fig. 3) even for participants taking or not taking chronic OCS.

\section{Airway T2 characteristics of asthma phenotypes}

In addition to the clinical and clinical biomarker 'clustering' variables assessed, plus sputum differential cell counts, biopsy gene expression was available in 81 of 156 subjects included in the ADEPT clustering. An active airway T2 phenotype was defined as the observed interleukin (IL)-13 activity in endobronchial samples, indicated by the gene expression of IL-13-inducible genes. For the ADEPT study, this was specifically evaluated by gene expression of CCL26 in endobronchial biopsies. Despite having the $2^{\text {nd }}$ lowest levels of FENO, blood eosinophils, and sputum eosinophils, Phenotype A1 had the highest proportion of participants (56\%) who had high CCL26 gene expression in their endobronchial biopsies (CCL26-high asthmatics defined as beyond the $95^{\text {th }}$ percentile of healthy control distribution; Additional file 1: Section S5), followed by Phenotype A2, which had slightly fewer (41\%) CCL26-high participants (Table 1). Consistent with being the least eosinophilic, Phenotype A3 had the least (16\%) CCL26high participants (Table 1). Phenotype A4 was intermediate, with 32\% CCL26-high participants. A similar pattern among the phenotypes was observed for POSTN gene expression as an additional indicator of T2 phenotype (data not shown).

Too few of the 82 U-BIOPRED asthma participants included in the clustering had endobronchial biopsy or brushings samples available for analysis $(n=16$ and 17, respectively). For the 77 of 397 U-BIOPRED participants classified to ADEPT clusters who had available data on microarray gene expression in epithelial brushings, the T2 distributions (defined by IL-13 in vitro stimulation signature enrichment) were similar to those observed for ADEPT participants despite the differences in tissue and indicators for defining T2 status (Data not shown and Additional file 1: Figure S4), with Phenotypes US2 and US4 having the highest proportions of T2-high participants (38\%) and Phenotype US3 having the fewest (6\%). Only the U-BIOPRED Phenotype US1 had lower proportions of T2-high participants (21\%) compared to Phenotype A1 (56\%), perhaps a consequence of ICS use in the U-BIOPRED but not ADEPT asthmatics in Phenotype A1. 


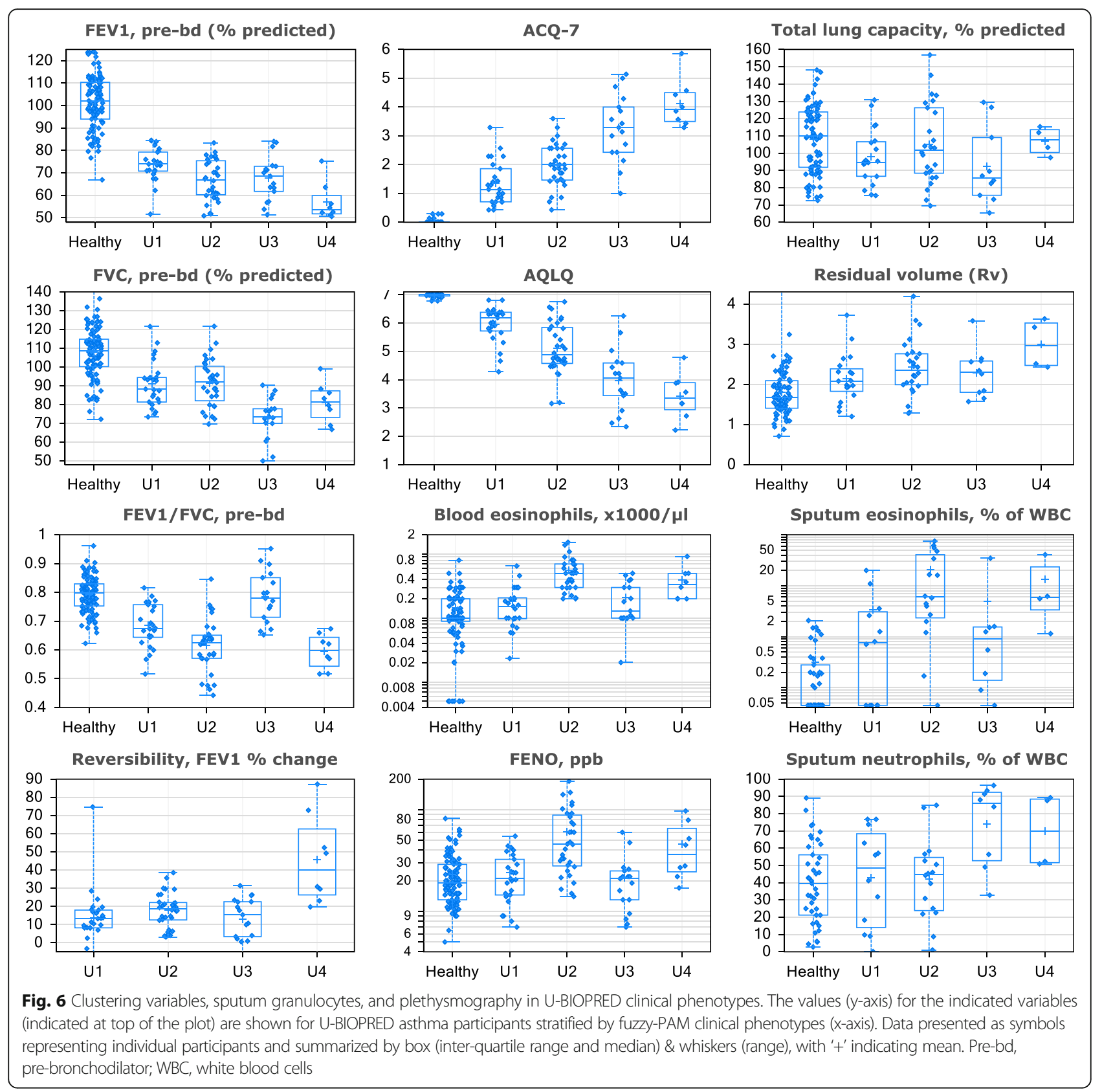

\section{Discussion}

To our knowledge, this is the first study to identify reproducible phenotypes of asthma using data from two entirely independent cohorts of asthmatic patients. The four observed phenotypes have clinical relevance, representing patient subtypes that are commonly encountered in clinical practice, namely mild, well-controlled asthmatics, moderately-controlled asthmatics with severe AHR, asthmatic with less reversible airways disease, and severe asthmatics. The large size of the study, enrolling participants from across the EU, US and Canada, and the use of clinical variables that can be obtained easily in any pulmonary center provides a valuable novel methodological tool for asthma research.

Initial clustering of the ADEPT study dataset resulted in four phenotypes that were robust to perturbation and generally stable over one year. These phenotypes were further validated in a subset of the U-BIOPRED cohort dataset, where four remarkably analogous phenotypes were observed. Importantly, differential biology across phenotypes was observed which will help develop tailored therapeutic options. Of relevance to current novel 
Table 3 Clinical, biomarker, and demographic associations among the U-BIOPRED adult asthma clinical clusters

\begin{tabular}{|c|c|c|c|c|c|c|}
\hline Variable $^{a}$ & $\begin{array}{l}\text { Healthy } \\
(n=101)\end{array}$ & $\begin{array}{l}\text { Cluster US1 } \\
(n=25)\end{array}$ & $\begin{array}{l}\text { Cluster US2 } \\
(n=32)\end{array}$ & $\begin{array}{l}\text { Cluster US3 } \\
(n=17)\end{array}$ & $\begin{array}{l}\text { Cluster US4 } \\
(n=8)\end{array}$ & $P$-value \\
\hline \multicolumn{7}{|l|}{ Mean [Geo.Mean] \pm SD } \\
\hline FEV1, \% predicted (pre-bd) & $101.8 \pm 12.9$ & $73.7 \pm 7.3$ & $66.6 \pm 9.0$ & $67.6 \pm 9.9$ & $57.0 \pm 8.5$ & $\begin{array}{l}8.8 \\
\times 10^{-5}\end{array}$ \\
\hline FVC, \% predicted (pre-bd) & $107.8 \pm 13.4$ & $90.9 \pm 12.4$ & $91.5 \pm 12.6$ & $72.9 \pm 11.5$ & $81.2 \pm 10.5$ & $\begin{array}{l}6.1 \\
\times 10^{-6}\end{array}$ \\
\hline FEV1/FVC (pre-bd) & $0.79 \pm 0.06$ & $0.68 \pm 0.08$ & $0.62 \pm 0.09$ & $0.79 \pm 0.09$ & $0.60 \pm 0.06$ & $<10^{-6}$ \\
\hline $\begin{array}{l}\text { bd reversibility, \% change } \\
\text { FEV1 }\end{array}$ & na & $13.7 \pm 16.7$ & $17.9 \pm 9.3$ & $12.9 \pm 12.5$ & $45.6 \pm 24.5$ & $\begin{array}{l}3.1 \\
x 10^{-6}\end{array}$ \\
\hline FENO, ppb [Geo.Mean] & $19.9+14.2 /-8.3$ & $20.8+16.0 /-9.1$ & $47.4+49.8 /-24.3$ & $18.1+15.0 /-8.2$ & $38.8+33.4 /-17.9$ & $\begin{array}{l}1.6 \\
\times 10^{-6}\end{array}$ \\
\hline ACQ7 & $0.0 \pm 0.1$ & $1.4 \pm 0.7$ & $2.0 \pm 0.7$ & $3.3 \pm 1.2$ & $4.1 \pm 0.8$ & $<10^{-6}$ \\
\hline AQLQ & $7.0 \pm 0.1$ & $6.0 \pm 0.6$ & $5.1 \pm 0.9$ & $4.0 \pm 1.1$ & $3.4 \pm 0.8$ & $<10^{-6}$ \\
\hline $\begin{array}{l}\text { Blood eosinophils, x1000/ul } \\
\text { [Geo.Mean] }\end{array}$ & $0.098+0.180 /-0.063$ & $0.143+0.155 /-0.075$ & $0.484+0.356 /-0.205$ & $0.161+0.204 /-0.090$ & $0.345+0.254 /-0.146$ & $<10^{-6}$ \\
\hline $\begin{array}{l}\text { Blood neutrophils, ×1000/ul } \\
\text { [Geo.Mean] }\end{array}$ & $3.23+1.67 /-1.10$ & $3.77+1.58 /-1.11$ & $4.09+1.33 /-1.00$ & $4.57+2.13 /-1.45$ & $1.84 \pm 0.49$ & 0.2128 \\
\hline $\begin{array}{l}\text { Blood WBC, } \times 1000 / u l \\
\text { [Geo.Mean] }\end{array}$ & $5.64+2.04 /-1.50$ & $6.41+1.62 /-1.29$ & $7.39+1.79 /-1.44$ & $7.83+2.24 /-1.74$ & $2.71 \pm 0.36$ & 0.0236 \\
\hline $\begin{array}{l}\text { Sputum neutrophils, \% of } \\
\text { WBC }^{b}\end{array}$ & $30.8+40.3 /-17.4$ & $42.8 \pm 28.3$ & $42.0 \pm 23.5$ & $73.9 \pm 24.2$ & $70.0 \pm 21.3$ & 0.0129 \\
\hline $\begin{array}{l}\text { Sputum eosinophils, \% of } \\
\text { WBC [Geo.Mean] }{ }^{\mathrm{b}}\end{array}$ & $0.2+0.3 /-0.1$ & $0.5+4.7 /-0.5$ & $6.1+44.5 /-5.4$ & $0.7+4.8 /-0.6$ & $6.3+21.0 /-4.9$ & 0.0123 \\
\hline IgE Total (IU/ml) [Geo.Mean] & $25+87 /-19$ & $121.1+377.2 /-91.7$ & $245.0+1,005.0 /-197.0$ & $84.5+267.5 /-64.2$ & $179.6+295.1 /-111.7$ & 0.0940 \\
\hline Age & $39 \pm 13$ & $46 \pm 15$ & $49 \pm 16$ & $50 \pm 12$ & $44 \pm 15$ & 0.7000 \\
\hline Age of onset, years & na & $21 \pm 18$ & $26 \pm 19$ & $29 \pm 19$ & $14 \pm 12$ & 0.2028 \\
\hline Body Mass Index (kg/m2) & $25.3 \pm 3.6$ & $27.6 \pm 5.4$ & $27.6 \pm 5.8$ & $30.9 \pm 7.8$ & $27.1 \pm 4.8$ & 0.2408 \\
\hline \multicolumn{7}{|l|}{ Percent of clinical cluster group } \\
\hline $\begin{array}{l}\text { Severe / Non-severe asthma } \\
(\mathrm{N} / \mathrm{N})\end{array}$ & na & $11 / 14$ & $25 / 7$ & $16 / 1$ & $8 / 0$ & 0.0004 \\
\hline Male (\%) & $61 \%$ & $36 \%$ & $34 \%$ & $29 \%$ & $25 \%$ & 0.9276 \\
\hline $\begin{array}{l}\text { Atopic, Skin prick test or serum } \\
\text { IgE-RAST (\%) }\end{array}$ & $45 \%$ & $100 \%$ & $86 \%$ & $88 \%$ & $100 \%$ & 0.5816 \\
\hline $\begin{array}{l}\text { Sputum Pauci (Eos }<\% 3 \text {, } \\
\text { PMN }<60 \%)(\%)^{\mathrm{b}}\end{array}$ & $76 \%$ & $42 \%$ & $25 \%$ & $25 \%$ & $0 \%$ & 0.0496 \\
\hline $\begin{array}{l}\text { Sputum Neutr. (Eos }<3 \text {, } \\
\text { PMN }>=60 \%)(\%)^{b}\end{array}$ & $24 \%$ & $33 \%$ & $6 \%$ & $63 \%$ & $25 \%$ & \\
\hline $\begin{array}{l}\text { Sputum Mix (Eos > =3\%, } \\
\text { PMN }>=60 \%)(\%)^{b}\end{array}$ & $0 \%$ & $0 \%$ & $6 \%$ & $0 \%$ & $25 \%$ & \\
\hline $\begin{array}{l}\text { Sputum Eos. (Eos > =3\%, } \\
\text { PMN }<60 \%)(\%)^{b}\end{array}$ & $0 \%$ & $25 \%$ & $63 \%$ & $13 \%$ & $50 \%$ & \\
\hline FENO $\geq 35$ ppb (\%) & $18 \%$ & $24 \%$ & $59 \%$ & $12 \%$ & $50 \%$ & 0.0032 \\
\hline Blood eosinophils $\geq 300 /$ ul (\%) & $7 \%$ & $20 \%$ & $75 \%$ & $35 \%$ & $63 \%$ & 0.0003 \\
\hline $\begin{array}{l}\text { FENO } \geq 35 \text { ppb OR Blood eos. } \\
\geq 300 / \text { ul (\%) }\end{array}$ & $21 \%$ & $32 \%$ & $88 \%$ & $41 \%$ & $88 \%$ & $\begin{array}{l}3.3 \\
\times 10^{-5}\end{array}$ \\
\hline
\end{tabular}

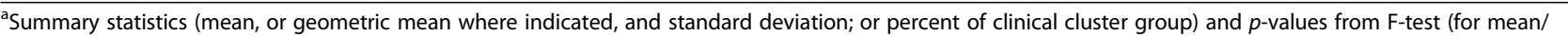
geometric mean statistics) or chi-square statistics for associations among the U-BIOPRED adult asthma clinical cluster groups are presented. The 8 clinical and clinical biomarker variables used in the clustering are presented first, followed by additional clinical, biomarker and demographic variable groupings

${ }^{\mathrm{b}}$ For sputum differentials, $n=12,16,8$, and 4 for the healthy control cohort and Groups A, B, and C, and D, respectively

Significant $p$ values are captured in bold 


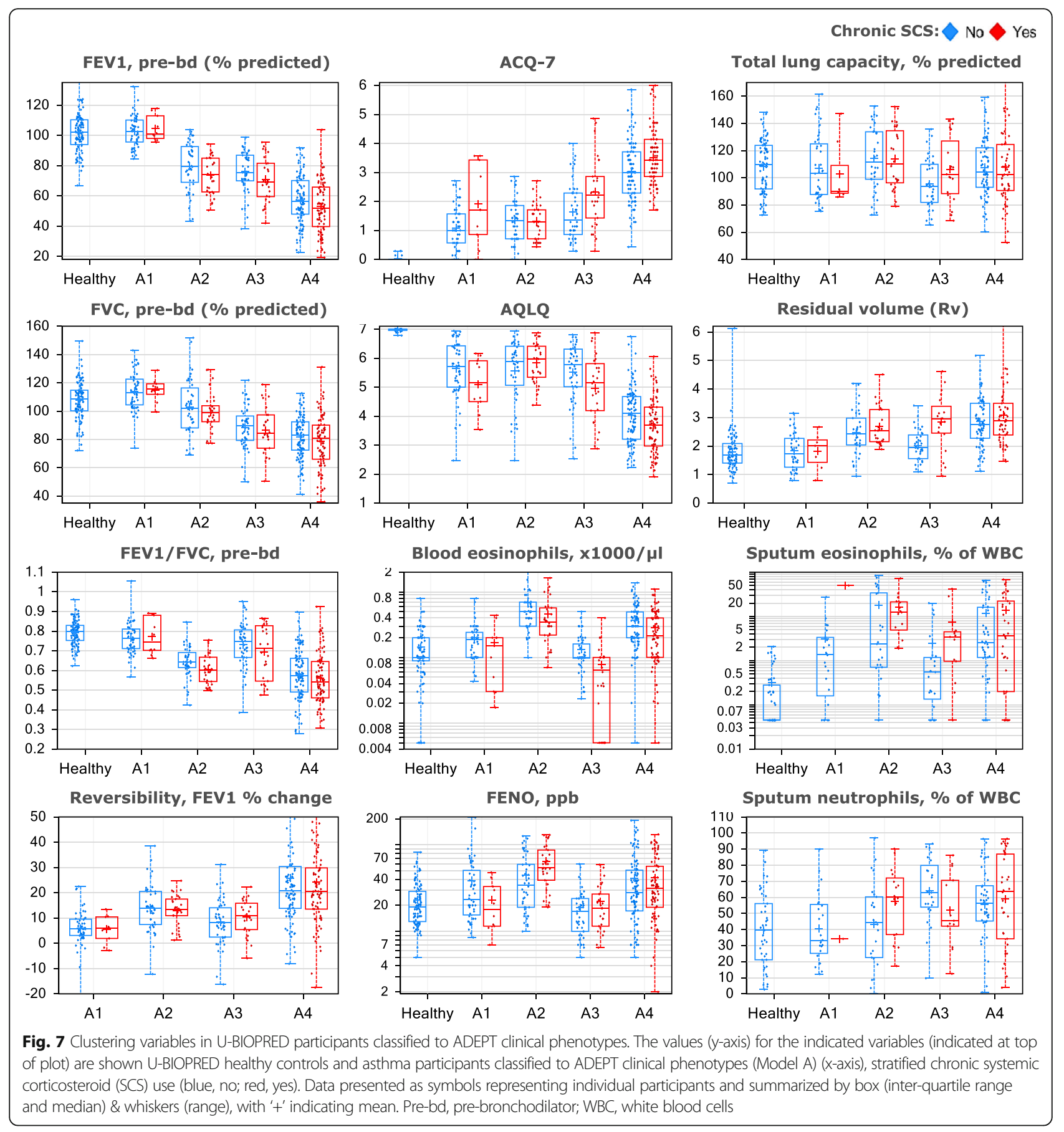

therapies that target $\mathrm{T} 2$ immune mechanisms, the phenotypes could be differentiated in respect of expression of gene sets that are characteristic of the pathobiology driven by the T2 cytokine, IL-13. Phenotype A1, was remarkably similar to Phenotype US1 from the U-BIOPRED validation set. The low degree of airway inflammation in A1 was commensurate with absence of a decision to start controller medications, while in the US1 phenotype, where all the asthmatics were on ICS, this suggested adequate suppression of airway inflammation and, consequently, good disease control. Of note, the A1 phenotype had the highest proportion of T2 high asthmatics even though their levels of exhaled NO, proposed as good biomarkers of T2 inflammation [20], were relatively low. This suggests that $\mathrm{T} 2$ inflammation, on its own, may not be a determinant of clinical severity. Given the good 
clinical characteristics of this phenotype, there seems to be minimal need for new therapies, although this phenotype could be targeted for disease interception.

The asthmatics in Phenotype A2, based on their ACQ7 scores (mean 1.1), were between the cut-off for good $(<0.75)$ and poor control $(>1.5)$, suggesting less than optimal control. With respect to underlying pathobiology, their gene expression profile was highly skewed towards T2 inflammation. Consistent with previous reports in mild, steroid-naive asthmatics [21], the high expression of IL-13 induced genes was associated with the highest eosinophilic profile amongst all four phenotypes. The preserved lung function and asthma control, but severe AHR may represent "brittle" asthma, a recognized patient phenotype. Given that these asthmatics were on regular treatment with corticosteroids, the high $\mathrm{T} 2$ profile suggests a significant degree of steroid insensitivity; therefore, treatment with biologics or other drugs targeting T2 inflammation might be indicated in this phenotype to achieve improved control.

Phenotype A3 had reduced lung capacity and lower BDR (compared to A2 and A4), which could suggest more extensive tissue remodeling. The asthmatics in this phenotype tended to be more neutrophilic and could, therefore, be viewed as having phenotypic similarities to COPD, with disease potentially driven more by infection than atopy. Consistent with the neutrophilic, rather than eosinophilic, nature of the disease, T2 gene expression was the lowest of all phenotypes. While not studied in ADEPT, the role of the microbiome in the airway or GI tract might be important, supporting evaluation of alternative anti-inflammatories e.g. macrolide antibiotics in this phenotype [22]. This phenotype has few therapeutic options at present so further study of the underlying mechanisms and how these impacts on clinical expression are needed.

Of all the observed phenotypes, Phenotype A4 was the most severe, with ACQ (mean 2.6) well above the cut-off for poor control and with $63 \%$ of participants classified as severe on enrollment. These asthmatics had the worse mean $\mathrm{FEV}_{1}(66 \%)$ and were severely hyper-responsive. Their sputum had a mixed granulocytic profile, with both neutrophil and eosinophil counts being high. Of importance, they had an airway T2-high profile, despite treatment with ICS, which could, like A2, represent relative steroid insensitivity. Patients with this profile are the commonest participants included in recent asthma studies for novel T2inflammation focused therapeutics, e.g. anti-IL-13 and anti- IL-5 monoclonal antibodies [23, 24]. These asthmatics had the second highest concentrations of FENO. Thus, those asthmatics in this phenotype with high levels of predictive biomarkers such as eosinophilia and FENO may well respond to therapies targeting IL-13 biology and/or eosinophilic inflammation
[23, 25]. Alternatively, high FENO concentrations could represent poor adherence to treatment with ICS [26].

There are many possible influences on stability of phenotypes such as allergen exposure, air pollution, as well as emergent changes in asthma medications, and viral infections. Thus, it is interesting that ADEPT participants remained relatively stable for their phenotype assignments throughout the 12-month duration of the study, suggesting that the phenotypes represent different disease driving biological mechanism but their specific combinations within a phenotype remain stable over time. Phenotype A4 was the least stable of the groups, and we would speculate that periods of reduced environmental triggers could improve control in this most severe group, resulting in a shift to Phenotype A2. Alternatively, improved responsiveness to inhaled steroids with resulting reduction in T2 inflammation (whether environmentally based or from improved adherence during the study) could result in a shift to Phenotype A3. However, the appropriate data to support such possibilities are not available and thus such explanations remain speculative.

This study makes valuable observations when comparing two entirely independent cohorts where participants are not matched a priori. Participants in the ADEPT study were selected on the basis of steroid therapy and significant obstruction $\left(\mathrm{FEV}_{1} \leq 80 \%\right.$ of predicted), in order to match standard interventional clinical trial enrollment criteria. At the same time, more restrictive limits were placed on other clinical parameters such as BMI $\left(\leq 32 \mathrm{~kg} / \mathrm{m}^{2}\right)$ and smoking asthmatics were excluded. U-BIOPRED had a much broader representation of the overall asthmatic population, including severe asthmatics on maintenance OCS treatment and much lower $\mathrm{FEV}_{1}$. Because of these differences, the U-BIOPRED primary validation set only included those participants most similar to those in ADEPT. Although $\mathrm{PC}_{20}$ data were not available for most of the U-BIOPRED participants, the four ADEPT phenotypes largely mapped to the resultant 4 U-BIOPRED phenotypes for clinical variables. However, when applying the ADEPT classification model to a larger subset of the adult participants in UBIOPRED cohort, the asthmatics on maintenance OCS and smoking-associated severe asthma also fitted well into the ADEPT phenotype classification structure, with similar relative distributions of the 8 clustering variables across the 4 classified ADEPT clinical phenotypes.

When considering the underlying biology, there was substantial homology between Phenotypes A1, A2, A3, and A4 and Phenotypes US1, US2, US3 and US4, respectively, with similar homology for most inflammatory variables. In comparison to Phenotype A2, the equivalent US2 phenotype from U-BIOPRED, had very similar bronchodilator responsiveness, albeit slightly worse prebronchodilator $\mathrm{FEV}_{1}$ (mean 66\%). The $\mathrm{T} 2$ profile in Phenotype US2 was similarly skewed towards T2. Both 
Phenotypes A3 and US3 shared features with COPD (lower BDR compared to A2 and A4, low FENO, and neutrophilia). However, despite relatively low eosinophilic inflammation in both A1 and US1, FENO and airway T2high status tended to be higher in A1 but low in US1, likely a consequence of ICS requirement in U-BIOPRED but not in ADEPT. This observation suggests that FENO levels may be more directly associated with lower airway T2 activity than eosinophilic inflammation per se. We have observed that the T2-high phenotype is a subset of a broader eosinophilic phenotype group in ADEPT and UBIOPRED studies [27], consistent with results from Choy et al. [28] in mild asthma (evaluating T2 phenotype status by POSTN, SERPINB2, and CLCA1 expression in endobronchial brushings).

T2 inflammation is a major characteristic defining the clusters, but mainly distinguishes Phenotype A3 (low T2 inflammation) from the other 3 phenotype groups. Phenotypes A1, A2, and A4 are then clinically distinguished by degree of asthma control and airflow limitations. Ideally T2 and eosinophilic inflammation would be established from airway mucosal and sputum sampling, but these are difficult to evaluate in a standard clinical setting. Blood eosinophils, FENO, and potentially serum proteins could serve as surrogates to establish these phenotypes, but alone are insufficient to define such. Combinations of the surrogates, as utilized in the classification of the phenotype groups, are needed for more accurate estimation of $\mathrm{T} 2$ and eosinophilic phenotypes.

The variables selected for clustering in this study were different from those in previous reports (Additional file 1: Table S1), applying variables readily measured in clinical practice and trials that represent the current clinical presentation of asthma, as opposed to demographic and historical aspects of the disease. Likewise, although gender and smoking history may be associated with disease, they do not directly represent the pathology of asthma. Posthoc perturbation analyses (not reported) assessing the impact of adding in additional demographic, historical, and treatment attributes (e.g., gender, age, atopy, asthma age-of-onset, BMI, ICS dose; 20 variables total), resulted in weaker clustering (based on distance metrics and cluster membership probabilities), with qualitatively similar clinical associations as reported for the ADEPT asthma phenotypes (65\% concordance of the most closely analogous clusters with the reported ADEPT asthma phenotypes). FENO was an influential variable in the clustering. Inclusion of FENO could potentially indicate steroid insensitivity and/or a basal non-inflammatory phenotype. Given that FENO and blood eosinophil levels were not higher in cluster A1 (mostly no ICS) compared to clusters 2 and 4 (low-medium and high ICS), FENO and blood eosinophil levels are not simply an indicator of ICS use. Compliance was not formally monitored in these studies.
However, suppression of B cell and T cell lineage gene expression in biopsies below levels in healthy controls and mild asthmatics (not taking ICS), and suppression of mast cell lineage gene expression below that in mild asthma (data not shown), suggests that most subjects in the moderate and severe asthma groups were compliant in taking ICS, at least around the time of biopsy. The fitting of the U-BIOPRED severe asthmatics on oral CS to the clusters furthers confidence that the cluster formations were not overly influenced by lack of compliance to steroids. Nevertheless, even in the best of circumstances, compliance can only be evaluated over the short-term and imperfectly in absence of inpatient-monitoring.

The strength of this study is represented by the independent, external validation and the longitudinal stability assessment. Nevertheless, there are also limitations. The actual prevalence of the clinical clusters cannot be directly estimated because participants were not randomly recruited from the general asthma population. ADEPT had strict inclusion/exclusion criteria to restrict enrollment to patients likely to be enrolled in interventional clinical studies. Thus morbidly obese participants, smoking participants and chronic OCS-treated participants are not represented in ADEPT. In contrast, U-BIOPRED did include smokers and chronic OCS treated patients, with almost $50 \%$ of asthmatics on chronic OCS therapy. These features in fact predominated in the distinction of the 4 clinical clusters that have been reported with the U-BIOPRED cohort [29] (Additional file 1: Section S12). Indeed, 4 clusters were identified, of which the first consisted of well-controlled mild-to-moderate asthmatics, while the 3 other clusters consisting of predominantly severe asthma patients were characterised by 2 clusters of chronic airflow obstruction, one with late-onset asthma in predominant smokers/ex-smokers with high BMI and the other in non-smokers with high OCS use; the fourth cluster consisted of predominantly obese female patients with uncontrolled asthma and increased exacerbations, but with normal lung function [29].

\section{Conclusion}

In summary, we have provided evidence for four phenotypes that are stable over time and are differentiated by both clinical severity, response to their prescribed treatment and the underlying T2 gene expression profile. Critically, these phenotypic groups were validated in an independent asthma cohort. Further extensive gene expression data and other 'omics' analyses remain available for more in-depth evaluation of molecular profiles associated with these newly defined clinical phenotypic groups. Focusing on the biology of each phenotype and understanding the unmet need will aid in developing tailored therapies. 


\section{Additional file}

Additional file 1: Table S1. List of clustering variables for several studies. Table S2. Clinical and demographic characteristics of ADEPT and U-BIOPRED severity cohorts. Table S3. Linear predictor function coefficients for classification to ADEPT-asthma baseline clinical clusters. Table S4. IL-13 in vitro stimulation (IVS) signature. Table S5. Plethysmography and PRO variable associations among the U-BIOPRED adult asthma clinical clusters. Figure S1. (A). Longitudinal ADEPT cluster classifications discordant with baseline Cluster A1. Figure S1. (B). Longitudinal ADEPT cluster classifications discordant with baseline Cluster A2. Figure S1. (C). Longitudinal ADEPT cluster classifications discordant with baseline Cluster A3. Figure S1. (D). Longitudinal ADEPT cluster classifications discordant with baseline Cluster A4. Figure S2. Characterization of selected subjects changing ADEPT cluster classifications. Figure S3. Characterization of selected subjects with stable ADEPT cluster classifications. Figure $\mathbf{S 4}$. IL-13 activity in U-BIOPRED participants classified to ADEPT clinical clusters. (XLSX $461 \mathrm{~kb})$

\section{Abbreviations}

ACQ7: Asthma control questionnaire 7 (include FEV1); ADEPT: Airways Disease Endotyping for Personalized Therapeutics; AHR: Airways hyperresponsiveness; AQLQ: Asthma quality of life questionnaire; BDR: Bronchodilator reversibility; bEOS: Blood eosinophils; CCL: C-C motif chemokine ligand; EBBX: Endobronchial biopsy; EOS: Eosinophils; EPBR: Epithelial brushings; FEF25-75: Forced expired flow averaged between $25-75 \%$ of the vital capacity; FENO: Fractional concentration of exhaled nitric oxide; FEV1: Forced expired volume in 1 second; FVC: Forced vital capacity; GSVA: Gene set variation analysis; HV: Healthy Volunteers; ICS: Inhaled corticosteroids; IFN: Interferon; IgE: Immunoglobulin E; IL: interleukin; IL-4R: Interleukin 4 receptor; IS: Induced sputum; IVS: In-vitro signature; LOQ: Limit of quantification; MAB: Monoclonal antibody; Mod-Sev: Moderate-severe asthma (combined); mRNA: Messenger ribonucleic acid; NHANES: National Health and Nutrition Examination Survey; PC20: The provocative concentration of methacholine resulting in a $20 \%$ or greater fall in the forced expired volume in $1 \mathrm{~s}$; PN: Predicted normal; RNA: Ribonucleic acid; SARP: Severe Asthma Research Program; SCS: Systemic corticosteroids; spEOS: Sputum eosinophils; SST: Serum separation tubes; Th2: T helper cell type 2; TLC: Total lung capacity; U-BIOPRED: Unbiased Biomarkers for the Prediction of Respiratory Disease Outcome Consortium

\section{Acknowledgements}

Keith Lasher and the Janssen Global Clinical Organization for management of the ADEPT clinical trial. The investigators who participated in the ADEPT study: I. Strambu, Arensia Exploratory Medicine, Sos. Viilor 90, Bucharest 050159, Romania; M. Laviolette, Institut Universitaire de Cardiologie et Pneumologie de Québec (IUCPQ), 2725, Chemin Ste-Foy, Québec G1V 4G5, Canada; D. Singh, Medicines Evaluation Unit, University Hospital of South Manchester Foundation Trust, University of Manchester, Southmoor Road Manchester M23 9QZ, UK; J. M. FitzGerald, Institute for Heart and Lung Health, The Lung Centre, 7th Floor, Gordon, Canada \& Leslie Diamond Health Care Centre, 2775 Laurel Street, Vancouver, BC V5Z 1 M9, Canada; S. Lam, Institute for Heart and Lung Health, The Lung Centre, 7th Floor, Gordon, Canada \& Leslie Diamond Health Care Centre, 2775 Laurel Street, Vancouver, BC V5Z 1 M9, Canada; S. Kelsen, Temple University Hospital, 3401 N. Broad Street, Philadelphia, PA 19140, USA; A. Eich, IKF Pneumologie Frankfurt, Institut für klinische Forschung Pneumologie, Clinical Research Centre Respiratory Diseases, Schaumainkai 101-103, Stresemannallee, 360596 Frankfurt, Germany; A. Ludwig-Sengpiel, KLB Gesundheitsforschung Lübeck GmbH, Sandstr. 18, 23552 Lübeck, Germany; G. C hupp, Yale School of Medicine, TAC 441, 300 Cedar Street, New Haven, CT 06520,USA; V. Backer, Department of Respiratory Medicine, Respiratory Research Unit, Bispebjerg University Hospital, Bispebjerg bakke 23, DK-2400 Copenhagen,NV, Denmark; C. Porsbjerg, Department of Respiratory Medicine, Respiratory Research Unit, Bispebjerg University Hospital, Bispebjerg bakke 23, DK-2400 Copenhagen,NV, Denmark; P. O. Girodet, Univ. Bordeaux, Centre de Recherche CardioThoracique de Bordeaux, U1045, CIC 1401, F-33000 Bordeaux, France; P. Berger, Univ. Bordeaux, Centre de Recherche Cardio-Thoracique de Bordeaux, U1045, CIC 1401, F-33000 Bordeaux, France; R. Leigh, Cumming School of Medicine, University of Calgary, 3280 Hospital Drive NW, Calgary, AB T2N 4Z6, Canada; J. Kline, Division of Pulmonary, Critical Care, and Occupational Medicine, University of lowa, W219B GH UIHC, 200 Hawkins Drive, lowa City,
IA 52242, USA; M. Dransfield, Division of Pulmonary, Allergy and Critical Care Medicine, University of Alabama at Birmingham \& Birmingham VA Medical Center, 422 THT, 1900 University Blvd, Birmingham, AL 35294, USA; W. Calhoun, 116 John Sealy Annex, University of Texas Medical Branch, 301 University Blvd, Galveston, TX 77555-0568, USA; A. Hussaini, Parexel International, Shelton Simmons (MD), 3001 S Hanover St \#7, Brooklyn, MD 21225, USA; S. Khatri, Department of Pulmonary and Critical Care, Cleveland Clinic, 9500 Euclid Avenue, Cleveland, OH 44195, USA; P. Chanez, Pneumologie, Aix Marseille University, APHM/ INSERM U1067, Chemin des Bourellys, 13015 Marseille, France. U-BIOPRED has been funded by a public-private grant from the Innovative Medicines Initiative (IMI) provided by the European Union (EU) and the European Federation of Pharmaceutical Industries and Associations. U-BIOPRED has been funded by a public-private grant from the Innovative Medicines Initiative (IMI) provided by the European Union (EU) and the European Federation of Pharmaceutical Industries and Associations. The members of the U-BIOPRED Study Group are as follows:

Adcock lan, National Heart and Lung Institute, Imperial College, London, UK; Adriaens Nora, Academic Medical Centre, University of Amsterdam, The Netherlands;

Ahmed Hassan, European Institute for Systems Biology and Medicine, CNRS-ENS-UCBL-INSERM, Lyon, France;

Aliprantis Antonios, Merck Research Laboratories, Boston, USA;

Alving Kjell, Dept Women's \& Children's Health, Uppsala University, Sweden

Auffray Charles, European Institute for Systems Biology and Medicine,

CNRS-ENS-UCBL-INSERM, Lyon, France;

Badorrek Philipp, Fraunhofer ITEM; Cornelia Faulenbach, Fraunhofer ITEM;

Bakke Per, Department of Clinical Science, University of Bergen, Bergen, Norway; Balgoma David, Centre for Allergy Research, Karolinska Institutet, Stockholm, Sweden

Bansal Aruna T., Acclarogen Ltd, St. John's Innovation Centre, Cambrigde, UK; Barber Clair, NIHR Southampton Respiratory Biomedical Research Unit and Clinical and Experimental Sciences, Southampton, UK:

Baribaud Frédéric, Janssen R\&D, USA;

Bautmans An, MSD, Brussels, BE

Behndig Annelie F., Dept of Public Health and Clinical Medicine, Umeå University, Umeå, Sweden;

Bel Elisabeth, Academic Medical Centre, University of Amsterdam, The Netherlands; Beleta Jorge, Almirall S.A., Barcelona, Spain;

Berglind Ann, Dept. Women's and Children's Health \& Centre for Allergy Research, Karolinska Institutet, Stockholm, Sweden

Berton Alix, AstraZeneca, Mölndal, Sweden

Bigler Jeannette, Previously at: Amgen Inc.;

Bisgaard Hans, COPSAC, Copenhagen Prospective Studies on Asthma in

Childhood, Herlev and Gentofte Hospital,

University of Copenhagen, Copenhagen, Denmark

Bochenek Grazyna, II Department of Internal Medicine, Jagiellonian University Medical College, Krakow, Poland;

Boedigheimer Michel J., Amgen Inc.;

Bøonnelykke Klaus, COPSAC, Copenhagen Prospective Studies on Asthma in Childhood, Herlev and Gentofte

Hospital, University of Copenhagen, Copenhagen, Denmark;

Brandsma Joost, University of Southampton, UK

Braun Armin, Fraunhofer Institute for Toxicology and Experimental

Medicine, Hannover, Germany;

Brinkman Paul, Academic Medical Centre, University of Amsterdam, The Netherlands;

Burg Dominic, Centre for Proteomic Research, Institute for Life Sciences, University of Southampton, UK

Campagna Davide, Department of Clinical and Experimental Medicine,

University of Catania, Italy;

Carayannopoulos Leon, MSD, USA;

Caruso Massimo, Dept. Clinical and Experimental Medicine, University of Catania, Italy;

Carvalho da Purificação Rocha João Pedro, Royal Brompton and Harefield NHS Foundation Trust, UK;

Chaiboonchoe Amphun, European Institute for Systems Biology and

Medicine, CNRS-ENS-UCBL-INSERM, CIRI-UMR5308, Lyon, France;

Chaleckis Romanas, Centre of Allergy Research, Karolinska Institutet,

Stockholm, Sweden

Chanez Pascal, Assistance publique des Hôpitaux de Marseille - Clinique des bronches, allergies et sommeil, Aix Marseille Université, Marseille France 
Chung Kian F., National Heart and Lung Institute, Imperial College, London, UK:

Coleman Courtney, Asthma UK, London, UK;

Compton Chris, Respiratory Therapeutic Unit, GSK, UK

Corfield Julie, Areteva R\&D, Nottingham, UK;

D'Amico Arnaldo, University of Rome 'Tor Vergata', Rome Italy;

Dahlen Barbro, Karolinska University Hospital \& Centre for Allergy

Research, Karolinska Institutet, Stockholm, Sweden

Dahlén Sven-Erik, Centre for Allergy Research, Karolinska Institutet, Stockholm, Sweden

De Alba Jorge, Almirall S.A., Barcelona, Spain;

de Boer Pim, Longfonds, Amersfoort, The Netherlands;

De Lepeleire Inge, MSD, Brussels, BE

De Meulder Betrand, European Institute for Systems Biology and Medicine,

CNRS-ENS-UCBL-INSERM, Lyon, France;

Dekker Tamara, Academic Medical Centre, University of Amsterdam,

The Netherlands;

Delin Ingrid, Centre for Allergy Research, Karolinska Institutet, Stockholm,

Sweden

Dennison Patrick, NIHR Southampton Respiratory Biomedical Research Unit, Clinical and Experimental Sciences, NIHR-Wellcome Trust Clinical Research Facility, Faculty of Medicine, University of Southampton, UK; Dijkhuis Annemiek, Academic Medical Centre, University of Amsterdam, The Netherlands;

Djukanovic Ratko, NIHR Southampton Respiratory Biomedical Research Unit and Clinical and Experimental Sciences, Southampton, UK;

Draper Aleksandra, BioSci Consulting, Maasmechelen, Belgium;

Edwards Jessica, Asthma UK, London, UK;

Emma Rosalia, Department of Clinical and Experimental Medicine, University

of Catania, Italy;

Ericsson Magnus, Karolinska University Hospital, Stockholm, Sweden

Erpenbeck Veit, Translational Medicine, Respiratory Profiling, Novartis Institutes for Biomedical Research, Basel, Switzerland;

Erzen Damijan, Boehringer Ingelheim Pharma GmbH \& Co. KG; Biberach, Germany

Fichtner Klaus, Boehringer Ingelheim Pharma GmbH \& Co. KG; Biberach, Germany

Fitch Neil, BioSci Consulting, Maasmechelen, Belgium;

Fleming Louis J., National Heart and Lung Institute, Imperial College,

London, UK

Flood Breda, Asthma UK, UK

Fowler Stephen J., Centre for Respiratory Medicine and Allergy, Institute of Inflammation and Repair, University of Manchester and University Hospital of South Manchester, Manchester Academic Health Sciences Centre, Manchester, United Kingdom

Frey Urs, University Children's Hospital, Basel, Switzerland; Gahlemann Martina, Boehringer Ingelheim (Schweiz) GmbH, Switzerland; Galffy Gabriella, Semmelweis University, Budapest, Hungary; Gallart Hector, Centre for Allergy Research, Karolinska Institutet, Stockholm, Sweden

Garrett Trevor, BioSci Consulting, Maasmechelen, Belgium;

Geiser Thomas, Department of Respiratory Medicine, University Hospital Bern, Switzerland;

Gent Julaiha, Royal Brompton and Harefield NHS Foundation Trust, UK; Gerhardsson de Verdier Maria, AstraZeneca; Molndal, Sweden;

Gibeon David, National Heart and Lung Institute, Imperial College, UK; Gomez Cristina, Centre for Allergy Research, Karolinska Institutet, Stockholm, Sweden

Gove Kerry, NIHR Southampton Respiratory Biomedical Research Unit and Clinical and Experimental Sciences, Southampton, UK;

Gozzard Neil, UCB, UK;

Guo Yi-ke, Data Science Institute, Imperial College, UK;

Hashimoto Simone, Academic Medical Centre, University of Amsterdam, The Netherlands;

Haughney John, International Primary Care Respiratory Group, Aberdeen, Scotland;

Hedlin Gunilla, Dept. Women's and Children's Health \& Centre for Allergy Research, Karolinska Institutet, Stockholm, Sweden;

Hekking Pieter-Paul, Academic Medical Centre, University of Amsterdam, The Netherlands;
Henriksson Elisabeth, Karolinska University Hospital \& Karolinska Institutet, Stockholm, Sweden

Hewitt Lorraine, NIHR Southampton Respiratory Biomedical Research Unit, Southampton, UK

Higgenbottam Tim, Allergy Therapeutics, West Sussex, UK;

Hoda Uruj, Imperial College, UK;

Hohlfeld Jens, Fraunhofer Institute for Toxicology and Experimental

Medicine, Hannover, Germany;

Holweg Cecile, Respiratory and Allergy Diseases, Genentech,

San Francisco CA;

Horvath Ildiko, Semmelweis University, Budapest, Hungary;

Howarth Peter, NIHR Southampton Respiratory Biomedical Research Unit, Clinical and Experimental Sciences and Human Development and Health,

Southampton, UK;

Hu Richard, Amgen Inc:;

Hu Sile, National Heart and Lung Institute, Imperial College, UK;

Hu Xugang, Amgen Inc.:

Hudson Val, Asthma UK, London, UK;

James Anna J., Centre for Allergy Research, Karolinska Institutet,

Stockholm, Sweden;

Kamphuis Juliette, Longfonds, Amersfoort, The Netherlands;

Kennington Erika J., Asthma UK, London, UK:

Kerry Dyson, CromSource, Stirling UK;

Klüglich Matthias, Boehringer Ingelheim Pharma GmbH \& Co. KG; Biberach, Germany

Knobel Hugo, Philips Research Laboratories, Eindhoven, The Netherlands;

Knowles Richard, Arachos Pharma, UK;

Knox Alan, Respiratory Research Unit, University of Nottingham, UK:

Kolmert Johan, Centre for Allergy Research, Karolinska Institutet, Stockholm,

Sweden

Konradsen Jon, Dept. Women's and Children's Health \& Centre for Allergy

Research, Karolinska Institutet, Stockholm, Sweden

Kots Maxim, Chiesi Pharmaceuticals, SPA, Parma, Italy;

Krueger Linn, University Children's Hospital Bern, Switzerland

Krug Norbert, Fraunhofer Institute for Toxicology and Experimental Medicine, Hannover, Germany:

Kuo Scott, National Heart and Lung Institute, Imperial College, UK;

Kupczyk Maciej, Centre for Allergy Research, Karolinska Institutet, Stockholm,

Sweden

Lambrecht Bart, University of Gent, Gent, Belgium;

Lantz Ann-Sofie, Karolinska University Hospital \& Centre for Allergy Research, Karolinska Institutet, Stockholm, Sweden;

Larsson Lars, AstraZeneca, Mohlndal, Sweden;

Lazarinis Nikos, Karolinska University Hospital \& Karolinska Institutet,

Stockholm, Sweden

Lefaudeux Diane, European Institute for Systems Biology and Medicine,

CNRS-ENS-UCBL-INSERM, Lyon, France;

Lone-Latif Saeeda, Academic Medical Centre, University of Amsterdam,

The Netherlands;

Loza Matthew J., Janssen R\&D, USA;

Lutter Rene, Academic Medical Centre, University of Amsterdam, The

Netherlands;

Marouzet Lisa, NIHR Southampton Respiratory Biomedical Research Unit,

Southampton, UK

Martin Jane, NIHR Southampton Respiratory Biomedical Research Unit,

Southampton, UK

Masefield Sarah, European Lung Foundation, Sheffield, UK;

Mathon Caroline, Centre of Allergy Research, Karolinska Institutet,

Stockholm, Sweden

Matthews John G., Respiratory and Allergy Diseases, Genentech, San

Francisco CA, USA;

Mazein Alexander, European Institute for Systems Biology and Medicine,

CNRS-ENS-UCBL-INSERM, CIRI-UMR5308, Lyon, France;

Meah Sally, National Heart and Lung Institute, Imperial College, UK;

Meiser Andrea, National Heart and Lung Institute, Imperial College, UK;

Menzies-Gow Andrew, Royal Brompton and Harefield NHS Foundation Trust, UK;

Metcalf Leanne, Previously at: Asthma UK, London, UK;

Middelveld Roelinde, Centre for Allergy Research, Karolinska Institutet,

Stockholm, Sweden

Mikus Maria, Science for Life Laboratory \& The Royal Institute of Technology,

Stockholm, Sweden; 
Miralpeix Montse, Almirall, Barcelona, Spain;

Monk Philip, Synairgen Research Ltd, Southampton, UK:

Montuschi Paolo, Università Cattolica del Sacro Cuore, Italy;

Mores Nadia, Università Cattolica del Sacro Cuore, Italy;

Murray Clare S., Centre for Respiratory Medicine and Allergy, Institute of Inflammation and Repair, University of Manchester and University Hospital of South Manchester, Manchester Academic Health Sciences Centre, Manchester, United Kingdom

Musial Jacek, II Department of Internal Medicine, Jagiellonian University Medical College, Krakow, Poland;

Myles David, Respiratory Therapeutic Unit, GSK, UK;

Naz Shama, Centre for Allergy Research, Karolinska Institutet, Stockholm,

Sweden

Nething Katja, Boehringer Ingelheim Pharma GmbH \& Co. KG; Biberach,

Germany

Nicholas Ben, University of Southampton, UK

Nihlen Ulf, AstraZeneca; Molndal, Sweden;

Nilsson Peter, Science for Life Laboratory \& The Royal Institute of Technology,

Stockholm, Sweden;

Nordlund Björn, Dept. Women's and Children's Health \& Centre for Allergy

Research, Karolinska Institutet, Stockholm, Sweden

Östling Jörgen, AstraZeneca, Mölndal, Sweden;

Pacino Antonio, Lega Italiano Anti Fumo, Catania, Italy;

Pahus Laurie, Assistance publique des Hôpitaux de Marseille, Clinique des bronches, allergies et sommeil

Espace Éthique Méditerranéen, Aix-Marseille Université, Marseille, France;

Palkonen Susanna, European Federation of Allergy and Airways Diseases

Patient's Associations, Brussels, Belgium.

Pandis loannis, Data Science Institute, Imperial College, London, UK

Pavlidis Stelios, National Heart and Lung Institute, Imperial College, UK

Pennazza Giorgio, University of Rome 'Tor Vergata', Rome Italy;

Petrén Anne, Centre for Allergy Research, Karolinska Institutet, Stockholm,

Sweden

Pink Sandy, NIHR Southampton Respiratory Biomedical Research Unit,

Southampton, UK

Postle Anthony, University of Southampton, UK

Powel Pippa, European Lung Foundation, Sheffield, UK

Rahman-Amin Malayka, Previously at: Asthma UK, London, UK;

Rao Navin, Janssen R\&D, USA;

Ravanetti Lara, Academic Medical Centre, University of Amsterdam, The

Netherlands;

Ray Emma, NIHR Southampton Respiratory Biomedical Research Unit,

Southampton, UK

Reinke Stacey, Centre for Allergy Research, Karolinska Institutet, Stockholm,

Sweden

Reynolds Leanne, Previously at: Asthma UK, London, UK;

Riemann Kathrin, Boehringer Ingelheim Pharma GmbH \& Co. KG; Biberach,

Germany

Riley John, Respiratory Therapeutic Unit, GSK, UK:

Robberechts Martine, MSD, Brussels, BE

Roberts Amanda, Asthma UK, UK;

Roberts Graham, NIHR Southampton Respiratory Biomedical Research Unit, Clinical and Experimental Sciences and Human Development and Health,

Southampton, UK:

Rossios Christos, National Heart and Lung Institute, Imperial College, UK;

Rowe Anthony, Janssen R\&D, UK

Russell Kirsty, National Heart and Lung Institute, Imperial College, UK;

Rutgers Michael, Longfonds, Amersfoort, The Netherlands;

Sandström Thomas, Dept of Public Health and Clinical Medicine, Umeå

University, Umeå, Sweden;

Santini Giuseppe, Università Cattolica del Sacro Cuore, Italy;

Santoninco Marco, University of Rome 'Tor Vergata', Rome Italy;

Schoelch Corinna, Boehringer Ingelheim Pharma GmbH \& Co. KG, Biberach,

Germany

Schofield James P. R., Centre for Proteomic Research, Institute for Life

Sciences, University of Southampton, UK

Seibold Wolfgang, Boehringer Ingelheim Pharma GmbH, Biberach, Germany

Shaw Dominick E., Respiratory Research Unit, University of Nottingham, UK;

Sigmund Ralf, Boehringer Ingelheim Pharma GmbH \& Co. KG; Biberach, Germany

Singer Florian, University Children's Hospital, Zurich, Switzerland;
Sjödin Marcus, Centre for Allergy Research, Karolinska Institutet, Stockholm, Sweden

Skipp Paul J., Centre for Proteomic Research, Institute for Life Sciences, University of Southampton, UK

Smids Barbara, Academic Medical Centre, University of Amsterdam, The Netherlands;

Smith Caroline, NIHR Southampton Respiratory Biomedical Research Unit,

Southampton, UK

Smith Jessica, Asthma UK, London, UK;

Smith Katherine M., University of Nottingham, UK;

Söderman Päivi, Dept. Women's and Children's Health, Karolinska Institutet,

Stockholm, Sweden

Sogbesan Adesimbo, Royal Brompton and Harefield NHS Foundation Trust, UK;

Sousa Ana R., Respiratory Therapeutic Unit, GSK, UK;

Staykova Doroteya, University of Southampton, UK

Sterk Peter J., Academic Medical Centre, University of Amsterdam, The Netherlands;

Strandberg Karin, Karolinska University Hospital \& Karolinska Institutet, Stockholm, Sweden

Sun Kai, National Heart and Lung Institute, Imperial College, UK;

Supple David, Asthma UK, UK.

Szentkereszty Marton, Semmelweis University, Budapest, Hungary;

Tamasi Lilla, Semmelweis University, Budapest, Hungary;

Tariq Kamran, NIHR Southampton Respiratory Biomedical Research Unit,

Clinical and Experimental Sciences, NIHR-Wellcome Trust Clinical Research Fa-

cility, Faculty of Medicine, University of Southampton, UK;

Thörngren John-Olof, Karolinska University Hospital, Sweden

Thornton Bob, MSD, USA;

Thorsen Jonathan, COPSAC, Copenhagen Prospective Studies on Asthma in

Childhood, Herlev and Gentofte

Hospital, University of Copenhagen, Copenhagen, Denmark;

Valente Salvatore, Università Cattolica del Sacro Cuore, Italy;

van Aalderen Wim, Academic Medical Centre, University of Amsterdam, The

Netherlands;

van de Pol Marianne, Academic Medical Centre, University of Amsterdam,

The Netherlands;

van Drunen Kees, Academic Medical Centre, University of Amsterdam, The

Netherlands;

van Geest Marleen, AstraZeneca, Mölndal, Sweden;

Versnel Jenny, Previously at: Asthma UK, London, UK

Vestbo Jorgen, Centre for Respiratory Medicine and Allergy, Institute of

Inflammation and Repair, University of Manchester and University Hospital of

South Manchester, Manchester Academic Health Sciences Centre,

Manchester, United Kingdom

Vink Anton, Philips Research Laboratories, Eindhoven, The Netherlands;

Vissing Nadja, COPSAC, Copenhagen Prospective Studies on Asthma in

Childhood, Herlev and Gentofte Hospital,

University of Copenhagen, Copenhagen, Denmark;

von Garnier Christophe, University Hospital Bern, Switzerland;

Wagener Ariane, Academic Medical Centre, University of Amsterdam, The Netherlands;

Wagers Scott, BioSci Consulting, Maasmechelen, Belgium;

Wald Frans, Boehringer Ingelheim Pharma GmbH \& Co. KG, Biberach,

Germany

Walker Samantha, Asthma UK, London, UK

Ward Jonathan, Histochemistry Research Unit, Faculty of Medicine, University

of Southampton, Southampton, UK;

Weiszhart Zsoka, Semmelweis University, Budapest, Hungary;

Wetzel Kristiane, Boehringer Ingelheim Pharma GmbH, Biberach, Germany

Wheelock Craig E., Centre for Allergy Research, Karolinska Institutet,

Stockholm, Sweden;

Wiegman Coen, National Heart and Lung Institute, Imperial College, UK:

Williams Siân, International Primary Care Respiratory Group, Aberdeen, Scotland;

Wilson Susan J., Histochemistry Research Unit, Faculty of Medicine, University

of Southampton, Southampton, UK;

Woodcock Ashley, Centre for Respiratory Medicine and Allergy, Institute of

Inflammation and Repair, University of Manchester and University Hospital of

South Manchester, Manchester Academic Health Sciences Centre,

Manchester, United Kingdom

Yang Xian, National Heart and Lung Institute, Imperial College, UK;

Yeyasingham Elizabeth, UK Clinical Operations, GSK, Stockley Park, UK; 
Yu Wen, Amgen Inc

Zetterquist Wilhelm, Dept. Women's and Children's Health \& Centre for Allergy Research, Karolinska Institutet, Stockholm, Sweden

Zwinderman Koos, Academic Medical Centre, University of Amsterdam, The Netherlands;

\section{Funding}

Janssen R\&D LLC (ADEPT), Innovative Medicine Initiative (U-BIOPRED).

\section{Availability of data and material}

For the review process we will make the ADEPT clinical data base available to the reviewers as well as a spreadsheet which allows to classify new patients. If published we will not as of now share the data because of our contractual agreement with the investigators of the study.

\section{Author's contributions}

Conception and design: MJL, RD, KFC, ESB, VSS, PES, PJS, FB; Acquisition of data: $\mathrm{DH}, \mathrm{KM}, \mathrm{PB}$; Analysis and Interpretation: MJL, RD, KFC, PB, ESB, PES, PJS, $F B$; Drafting the manuscript for important intellectual content: MJL, PES, RD, FB; Critical revisions: All authors; Approval of the final version: All authors.

\section{Competing interests}

MJL, DH, KM, PB, ESB, VSS and FB are current employees and shareholders of Janssen R\&D, LLC. Philip E. Silkoff is a former employee and shareholder of Janssen R\&D, LLC

\section{Consent for publication}

All participants provided written informed consent which included permission to publish the study findings in both ADEPT (NCT01274507) and UBIOPRED (NCT01982162)

\section{Ethics approval and consent to participate}

Both studies complied with the Principles of the Declaration of Helsinki and had ethics approval by the respective institutional review boards. All participants provided written informed consent. Both studies complied with the Principles of the Declaration of Helsinki and had ethics approval by the respective institutional review boards. All subjects provided written informed consent to participate (genomic DNA testing was optional). The clinicaltrials.gov identifier are NCT01274507 (ADEPT) and NCT01982162 (U-BIOPRED).

\section{Author details}

${ }^{1}$ Immunology Therapeutic Area, Janssen Research \& Development LLC, 1400 McKean Rd, Spring House 19477, USA. ${ }^{2} \mathrm{NIHR}$ Respiratory Biomedical Research Unit, Clinical and Experimental Sciences, Faculty of Medicine, University of Southampton, Southampton, UK. ${ }^{3}$ National Heart and Lung Institute, Imperial College \& Biomedical Research Unit, Royal Brompton \& Harefield NHS Trust, London, UK. ${ }^{4}$ Academic Medical Centre, University of Amsterdam, Amsterdam, The Netherlands.

Received: 20 August 2016 Accepted: 1 December 2016

Published online: 15 December 2016

\section{References}

1. Bel EH, Sousa A, Fleming $L$, et al. Diagnosis and definition of severe refractory asthma: an international consensus statement from the Innovative Medicine Initiative (IMI). Thorax. 2011;66:910-7.

2. Kupczyk $M$, ten Brinke $A$, et al. Frequent exacerbators-a distinct phenotype of severe asthma. Clin Exp Allergy. 2014;44(2):212-21.

3. Pavord ID, Korn S, et al. Mepolizumab for severe eosinophilic asthma (DREAM): a multicentre, double-blind, placebo-controlled trial. Lancet. 2012; 380(9842):651-9.

4. Bel EH, Wenzel SE, et al. Oral glucocorticoid-sparing effect of mepolizumab in eosinophilic asthma. N Engl J Med. 2014;371(13):1189-97.

5. Moore WC, Meyers DA, Wenzel SE, et al. Identification of asthma phenotypes using cluster analysis in the Severe Asthma Research Program. Am J Respir Crit Care Med. 2010;181:315-23.

6. Moore WC, Hastie AT, Li X, et al. Sputum neutrophil counts are associated with more severe asthma phenotypes using cluster analysis. J Allergy Clin Immunol. 2014;133:1557-63 e5.
7. Wu W, Bleecker E, Moore W, et al. Unsupervised phenotyping of Severe Asthma Research Program participants using expanded lung data. J Allergy Clin Immunol. 2014;133:1280-8.

8. Ortega H, Li H, Suruki R, Albers F, Gordon D, Yancey S. Cluster analysis and characterization of response to mepolizumab. A step closer to personalized medicine for patients with severe asthma. Ann Am Thorac Soc. 2014;11: 1011-7.

9. Woodruff PG, Boushey HA, et al. Genome-wide profiling identifies epithelial cell genes associated with asthma and with treatment response to corticosteroids. Proc Natl Acad Sci U S A. 2007;104(40):15858-63.

10. Silkoff PE, Strambu I, Laviolette M., et al. Asthma characteristics and biomarkers from the Airways Disease Endotyping for Personalized Therapeutics (ADEPT) ongitudinal profiling study. Respiratory Research; in press.

11. Shaw DE, Sousa AR, Fowler SJ, et al. Clinical and inflammatory characteristics of the European U-BIOPRED adult severe asthma cohort. Eur Respir J. 2015:46(5):1308-21.

12. Kaufman L, Rousseeuw PJ. Finding Groups in Data New York. NY, USA: John Wiley \& Sons; 1990.

13. Juniper EF, O'Byrne PM, Guyatt GH, Ferrie PJ, King DR. Development and validation of a questionnaire to measure asthma control. Eur Respir J. 1999; 14:902-7.

14. Juniper EF, Buist AS, Cox FM, Ferrie PJ, King DR. Validation of a standardized version of the Asthma Quality of Life Questionnaire. Chest. 1999;1 15:1265-70.

15. Haldar P, Pavord ID, Shaw DE, et al. Cluster analysis and clinical asthma phenotypes. Am J Respir Crit Care Med. 2008;178:218-24.

16. Kelly MM, Efthimiadis A, Hargreave FE. Induced sputum : selection method. Methods Mol Med. 2001;56:77-91.

17. Pavord ID, Pizzichini MM, Pizzichini $E$, et al. The use of induced sputum to investigate airway inflammation. Thorax. 1997;52(6):498-501.

18. Hanzelmann S, Castelo R, Guinney J. GSVA: gene set variation analysis for microarray and RNA-seq data. BMC Bioinformatics. 2013;14:7.

19. Merrett J. Merrett. Phadiatop-a novel lgE antibody screening test. TG Clin Allergy. 1987:17(5):409-16

20. Westerhof GA, Korevaar DA, et al. Biomarkers to identify sputum eosinophilia in different adult asthma phenotypes. Eur Respir J. 2015:46(3): 688-96.

21. Woodruff PG, Modrek B, et al. T-helper type 2-driven inflammation defines major subphenotypes of asthma. Am J Respir Crit Care Med. 2009;180(5): 388-95.

22. Simpson JL, Powell H, Boyle MJ, Scott RJ, Gibson PG. Clarithromycin targets neutrophilic airway inflammation in refractory asthma. Am J Respir Crit Care Med. 2008:177:148-55.

23. Corren J, Lemanske RF, Hanania NA, et al. Lebrikizumab treatment in adults with asthma. N Engl J Med. 2011:365:1088-98.

24. Ortega HG, Liu MC, Pavord ID, et al. Mepolizumab treatment in patients with severe eosinophilic asthma. N Engl J Med. 2014;371:1198-207.

25. Wenzel S, Ford L, Pearlman D, et al. Dupilumab in persistent asthma with elevated eosinophil levels. N Engl J Med. 2013;368(26):2455-66.

26. McNicholl DM, Stevenson M, et al. The utility of fractional exhaled nitric oxide suppression in the identification of nonadherence in difficult asthma. Am J Respir Crit Care Med. 2012;186(11):1102-8.

27. Silkoff PE, Laviolette M, Singh D, et al. Identification of Airway-mucosal Type 2 inflammation by Clinical Biomarkers in Asthma. JACl in press.

28. Choy DF, Hart KM, et al. TH2 and TH17 inflammatory pathways are reciprocally regulated in asthma. Sci Transl Med. 2015;7(301):301ra129.

29. Lefaudeux D, Chung KF, et al. Clustering analysis of clinical variables in U-BIOPRED adult asthma cohort. Eur Respir J. 2014:44·P225. 